\title{
A transformed stochastic Euler scheme for multidimensional transmission PDE
}

\author{
Pierre ÉTORÉ*,a, Miguel MARTINEZ ${ }^{\mathrm{b}}$ \\ ${ }^{a}$ Univ. Grenoble Alpes, CNRS, Inria, Grenoble INP, LJK \\ 700 Avenue Centrale \\ 38401 St Martin D'Hères, France \\ email:pierre.etore@univ-grenoble-alpes.fr \\ * Corresponding author \\ ${ }^{b}$ Univ. Gustave Eiffel, LAMA \\ 5, boulevard Descartes \\ 77454 Marne-la-Vallee cedex 2, France \\ email:miguel.martinez@u-pem.fr
}

\begin{abstract}
In this paper we consider multi-dimensional Partial Differential Equations (PDE) of parabolic type in divergence form. The coefficient matrix of the divergence operator is assumed to be discontinuous along some smooth interface. At this interface, the solution of the PDE presents a compatibility transmission condition of its co-normal derivatives (multi-dimensional diffraction problem). We prove an existence and uniqueness result for the solution and study its properties. In particular, we provide new estimates for the partial derivatives of the solution in the classical sense. We then construct a low complexity numerical Monte Carlo stochastic Euler scheme to approximate the solution of the PDE of interest. Using the afore mentioned estimates, we prove a convergence rate for our stochastic numerical method when the initial condition belongs to some iterated domain of the divergence form operator. Finally, we compare our results to classical deterministic numerical approximations and illustrate the accuracy of our method.
\end{abstract}

Keywords: Stochastic Differential Equations, Divergence Form Operators, Euler discretization scheme, Monte Carlo methods

\section{Introduction}

Given a finite time horizon $T$, a real valued function $x \mapsto u_{0}(x)$, and an elliptic symmetric matrix $x \mapsto a(x) \in \mathbb{R}^{d \times d}$, which is smooth except at the interface surface $\Gamma$ between subdomains $D_{ \pm}$of $\mathbb{R}^{d}\left(\Gamma=\bar{D}_{+} \cap \bar{D}_{-}\right)$, we consider the parabolic transmission (or diffraction) problem : find $u$ from $[0, T] \times \mathbb{R}^{d}$ to 
$\mathbb{R}$ satisfying

$$
\left\{\begin{array}{l}
\partial_{t} u(t, x)-\nabla \cdot\left(a(x) \nabla_{x} u(t, x)\right)=0, \quad \forall(t, x) \in(0, T] \times\left(\mathbb{R}^{d} \backslash \Gamma\right) \\
u(0, x)=u_{0}(x), \forall x \in \mathbb{R}^{d} \\
\left\langle a_{+} \nabla_{x} u_{+}(t, .)-a_{-} \nabla_{x} u_{-}(t, .), \nu\right\rangle=0 \text { and } u_{+}(t, .)=u_{-}(t, .) \text { along } \Gamma
\end{array}\right.
$$

(here $\nu$ denotes the unit vector field which is normal to $\Gamma$ and points to $D_{+}$).

The objective of this paper is to provide an efficient stochastic numerical resolution method for the solution of (1).

Parabolic equations involving $\nabla \cdot(a \nabla)$ have been a major preoccupation for mathematicians in the fifties and the sixties. We may cite the pioneering works of $[42,43],[7]$, and $[39,40,41]$ that prove the continuity of the solution of the Cauchy problem attached to $\nabla \cdot(a \nabla)$ and also the celebrated paper by [1], which gives upper and lower Gaussian estimate bounds for the fundamental solution of the operator $\nabla \cdot(a \nabla)$ (for a more modern perspective on evolution PDEs involving divergence form operators of type $\nabla \cdot(a \nabla)$ see also [33]). In these references assumptions on $a$ are very weak (it is assumed to be measurable, bounded and elliptic).

In the case where the matrix $a$ is assumed to be discontinuous along the regular boundaries of some nice disjoint connected open sets in $\mathbb{R}^{d}$, but smooth elsewhere, a refined analysis of the parabolic equation may be found in the monograph [23]. The authors interpret the parabolic equation as a diffraction problem with transmission conditions along the discontinuity boundaries, of the type of (1), and investigate the classical smoothness of its solution.

When the underlying space is one-dimensional and the discontinuity is at zero ( $\Gamma$ then reduces to the single point 0 ), the link between (1) and some asymmetric diffusion process $X$ is well known. More precisely one has that $u(t, x)=\mathbb{E}^{x}\left[u_{0}\left(X_{t}\right)\right]$ where $X$ is solution to the Stochastic Differential Equation (SDE) with local time

$$
d X_{t}=\sigma\left(X_{t}\right) d W_{t}+a^{\prime}\left(X_{t}\right) d t+\frac{a(0+)-a(0-)}{a(0+)+a(0-)} d L_{t}^{0}(X)
$$

where $\sigma^{2}=2 a$ and $a^{\prime}$ denotes a function that coincides with the first order derivative of $a$ outside zero, and can be set at any arbitrary value at zero. In (2) we have denoted $W$ a standard one-dimensional Brownian motion (B.m.), and $L_{t}^{0}(X)$ the symmetric local time of $X$ at time $t$. Under mild conditions (2) has a unique strong solution $X$, see [25]. Put in other words the operator $\nabla \cdot(a \nabla)$ appears as the infinitesimal generator of the diffusion $X$ solution of (2). Note that the local time term in (2) is a singular term that reflects the discontinuity of $a$ along $\Gamma=\{0\}$.

For a study of the one-dimensional case one may refer to the overview [26], [13], and the series of works $[35,36,37,29,9,10,11,12][28,8,16,27,3]$ where stochastic numerical schemes are presented. 
Then if one constructs a scheme $\bar{X}$ approaching $X$ (in law for example), we will have that $\mathbb{E}^{x}\left[u_{0}\left(\bar{X}_{t}\right)\right]$ approaches $u(t, x)$. This provides some stochastic numerical resolution method for the solution of (1). But none of the above cited works on the one-dimensional case can be directly adapted to the multidimensional case.

As a matter of fact, till now and up to our knowledge much fewer stochastic schemes have been proposed and studied to tackle the multidimensional case.

A natural idea would be to regularize the coefficient $a$ around the interface $\Gamma$, and then to perform a discretized stochastic scheme on the classical problem obtained by regularization (for smooth $a^{\varepsilon}$ the process $X$ in link with $\nabla \cdot\left(a^{\varepsilon} \nabla\right)$ is some Itô process with classical drift that can be approached by a standard Euler scheme). But then there is a balance to find between the regularization step and the discretization step. Such methods are less precise and less investigated (see [46] for some elements in this direction; see also some of our numerical results in Section 6).

For some results with no regularization procedure see [30], and [4] in the case of a diagonal coefficient matrix $a$ constant outside the discontinuity boundary $\Gamma$; see also [32], which attempts to interpret stochastically the deterministic Galerkin method using jump Markov Chains.

In this paper we will propose a stochastic numerical scheme that allows to treat the multidimensional case, when the matrix-valued diffusion coefficient $a$ is not necessarily diagonal, nor piecewise constant. We aim at treating the discontinuity of $a$ directly and use no regularization. The scheme we propose is of Euler type; it can be seen as en extension to the multidimensional case of the scheme studied in [37] (see some comments in Remark 4.4).

One of the difficulties of the multidimensional case is that the stochastic process $X$ naturally in link with the operator $\nabla \cdot(a \nabla)$ is more difficult to describe than in dimension one. One knows that the operator generates such a process $X$, which is Markov (see for instance [45]; on the Dirichlet form approach see [17], in particular Exercise 3.1.1 p. 111). We still have the link $u(t, x)=\mathbb{E}^{x}\left[u_{0}\left(X_{t}\right)\right]$. But the Itô dynamic of $X$ is difficult to establish and to exploit. In the companion paper [14] we have been able to prove (in the case $\mathbb{R}^{d}=\bar{D}_{+} \cup D_{-}$and $a$ has some smoothness in $D_{ \pm}$) that

$$
\begin{aligned}
X_{t}^{k}= & x_{k}+\int_{0}^{t} \sum_{j=1}^{d} \sigma_{k j}\left(X_{s}\right) d W_{s}^{j}+\int_{0}^{t} \sum_{j=1}^{d} \partial_{j} a_{k j}\left(X_{s}\right) \mathbb{I}_{X_{s} \in D} d s \\
& -\frac{1}{2} \int_{0}^{t} \gamma_{+, k}\left(X_{s}\right) d K_{s}+\frac{1}{2} \int_{0}^{t} \gamma_{-, k}\left(X_{s}\right) d K_{s}, \quad t \geq 0 .
\end{aligned}
$$

In this expression $W$ is a standard B.m., we have $\sigma \sigma^{*}=2 a$, the terms $\gamma_{ \pm, k}$ are some co-normal vectors to the surface $\Gamma=\bar{D}_{+} \cap \bar{D}_{-}$, and $K$ is the PCAF associated through the Revuz correspondence to the surface measure on $\Gamma$. Equation (3) is in some sense the multi-dimensional analog to (2), the singular term being now $-\frac{1}{2} \int_{0}^{t} \gamma_{+, k}\left(X_{s}\right) d K_{s}+\frac{1}{2} \int_{0}^{t} \gamma_{-, k}\left(X_{s}\right) d K_{s}$. 
However to infer from (3) an approximation scheme $\bar{X}$ for $X$ is not easy. In the afore mentioned works about the one-dimensional case, things are most often achieved with the help of Itô-Tanaka type formulas, that allow to manipulate SDEs with local time. In the multi-dimensional case we have not access to such a formula, and in addition we know less about the singular term $-\frac{1}{2} \int_{0}^{t} \gamma_{+, k}\left(X_{s}\right) d K_{s}+\frac{1}{2} \int_{0}^{t} \gamma_{-, k}\left(X_{s}\right) d K_{s}$ than we know about $L_{t}^{0}(X)$ in the onedimensional case.

Thus we are led, in the present paper, to contruct a stochastic scheme $\bar{X}$ such that $\mathbb{E}^{x}\left[u_{0}\left(\bar{X}_{t}\right)\right]$ approaches $u(t, x)$, but without seeking to approach $X$ by $\bar{X}$. The idea will be to perform a standard Euler scheme as long as $\bar{X}$ does not cross the boundary $\Gamma$. But when the scheme $\bar{X}$ crosses the boundary we will correct its position in a way that reflects the transmission condition in (1). The contribution of the paper are the following.

I) We will first study the PDE (1). We will show that, when the initial condition $u_{0}$ belongs to some iteration of the domain of $\nabla \cdot(a \nabla)$, this PDE has a classical solution. Then we prove the existence of global bounds for the partial derivatives of this solution (up to order four in the space variable) outside the discontinuity boundary $\Gamma$, for all strictly positive times (and not just for times $t$ satisfying $t \geq \varepsilon$ for some $\varepsilon>0$ ), and all the way up to the boundary (not only interior estimates). In our opinion these estimates are new (compared to [23]) and have an interest per se. These estimates will be needed to perform the convergence analysis of our scheme. The method we follow, in this PDE oriented part of the paper, is combining the Hille-Yosida theorem with results on elliptic transmission PDEs to be found in [38].

II) We propose our scheme and study its convergence rate. More precisely we prove that uniformly w.r.t. $x_{0}$

$$
\left|u\left(T, x_{0}\right)-\mathbb{E}^{x_{0}} u_{0}\left(\bar{X}_{T}^{n}\right)\right| \leq K \sqrt{h_{n}}
$$

where $h_{n}$ is the time step of our Euler scheme (see the precise assumptions and statement in Theorem 5.1). Note that this rate of convergence is naturally slower than in the classical smooth case (e.g. $[22,47,2])$ but is expected for these kind of transmission problems with boundary issues. In this respect our result is a slight improvement in comparison to the one obtained for the one-dimensional transmission case in [37] and [3].

The paper is organized as follows. In Section 2 we present the notations of the paper and our main assumptions. In Section 3 we define precisely and study the parabolic transmission problem (1), proving in particular an existence and uniqueness result for a classical solution, for which we get estimates for the space and time derivatives. In Section 4 we present our scheme, and in Section 5 we analyse its convergence. Section 6 is devoted to numerical experiments. 


\section{General notations and assumptions}

For two points $x, y \in \mathbb{R}^{d}$ we denote by $\langle x, y\rangle$ their scalar product $\langle x, y\rangle=$ $x^{*} y=\sum_{i=1}^{d} x_{i} y_{i}$. $\langle x, x\rangle$.

For a point $x \in \mathbb{R}^{d}$ we denote by $|x|$ its Euclidean norm i.e. $|x|^{2}=\sum_{i=1}^{d} x_{i}^{2}=$

We denote by $\left(e_{1}, \ldots, e_{d}\right)$ the usual orthonormal basis of $\mathbb{R}^{d}$.

For two metric spaces $E, F$ we will denote by $C(E ; F)$ the set of continuous functions from $E$ to $F$ and, for $1 \leq p \leq \infty$, by $C^{p}(E ; F)$ the set of functions in $C(E ; F)$ that are $p$ times differentiable with continuous derivatives.

We will denote by $C_{c}^{p}(E ; F)$ the set of functions in $C^{p}(E ; F)$ that have a compact support.

We will denote by $C_{b}^{p}(E ; F)$ the set of functions in $C^{p}(E ; F)$ that are continuous with bounded $p$ first derivatives $\left(C_{b}(E ; F)\right.$ denotes the set of functions in $C(E ; F)$ that are bounded).

If $F=\mathbb{R}$, we will sometimes simply write for instance $C(E)$ for $C(E ; \mathbb{R})$, for the sake of conciseness.

For any multi-index $\alpha=\left(i_{1}, \ldots, i_{d}\right) \in \mathbb{N}^{d}$ and $x=\left(x_{1}, \ldots, x_{d}\right) \in \mathbb{R}^{d}$, we note $x^{\alpha}$ the product $x_{1}^{i_{1}} \ldots x_{d}^{i_{d}}$ and $|\alpha|=i_{1}+\cdots+i_{d}$. So that for $u \in C^{|\alpha|}\left(\mathbb{R}^{d}\right)$ we will denote $\frac{\partial^{|\alpha|} u}{\partial x^{\alpha}}$, or in short $\partial^{\alpha} u$, the partial derivative $\partial_{x_{1}^{i_{1}}}^{i_{1}} \ldots \partial_{x_{d}^{i_{d}}}^{i_{d}} u$.

Let $U \subset \mathbb{R}^{d}$ an open subset. We will denote by $L^{2}(U)$ the set of square integrable functions from $U$ to $\mathbb{R}$ equipped with the usual norm and scalar product $\|\cdot\|_{L^{2}(U)}$ and $\langle\cdot, \cdot\rangle_{L^{2}(U)}$.

We denote $H^{1}(U)$ the usual Sobolev space $W^{1,2}(U)$, equipped with the usual norm $\|\cdot\|_{H^{1}(U)}$. We will denote by $D_{i} v$ the derivative in the distribution sense with respect to $x_{i}$ of $v \in L^{2}(U)$.

We recall that the space $H_{0}^{1}(U) \subset H^{1}(U)$ can be defined as $H_{0}^{1}(U)=$ $\overline{C_{c}^{\infty}(U ; \mathbb{R})}=\overline{C_{c}^{1}(U ; \mathbb{R})}$.

We denote $H^{-1}(U)$ the usual dual topological space of $H_{0}^{1}(U)$.

For $m \geq 2$, we denote $H^{m}(U)$ the usual Sobolev space $W^{m, 2}(U) \subset L^{2}(U)$ of functions having $m$ successive weak derivatives in $L^{2}(U)$.

The notion of a $C^{k}$ domain $U \subset \mathbb{R}^{d}$ with bounded boundary $\Gamma=\partial U$ is defined with the help of a system of local change of coordinates of class $C^{k}$ (see [38] Chap.3 pp. 89-90).

From now on we consider in the whole paper that $\mathbb{R}^{d}=\bar{D}_{+} \cup D_{-}$with $D_{+}$and $D_{-}$two open connected subdomains separated by a transmission boundary $\Gamma$ that is to say

$$
\Gamma=\bar{D}_{+} \cap \bar{D}_{-}
$$

(in addition we will denote $D=D_{+} \cup D_{-}=\mathbb{R}^{d} \backslash \Gamma \subset \mathbb{R}^{d}$ ).

By an assumption of type " $\Gamma$ is bounded and $C^{k}$ " we will mean that both $D_{+}$and $D_{-}$are $C^{k}$ domains, and that $\Gamma$ is bounded. Note that in that case we shall consider $D_{+}$(resp. $D_{-}$) as the interior (resp. exterior) domain. Note that $D_{-}$is then unbounded (although its boundary is bounded). 
Assume $\Gamma$ is bounded and $C^{2}$. We will denote $\gamma_{ \pm}: H^{1}\left(D_{ \pm}\right) \rightarrow H^{1 / 2}(\Gamma)$ the usual trace operators on $\Gamma$ and $H^{-1 / 2}(\Gamma)$ the dual space of $H^{1 / 2}(\Gamma)$ (see pp. 98-102 in [38]). We will denote $(u, v)_{\Gamma}$ the action of an element $u \in H^{-1 / 2}(\Gamma)$ on a element $v \in H^{1 / 2}(\Gamma)$ (this can be thought as a surface integral).

In the sequel we will frequently note $f_{ \pm}$the restrictions of a function $f$ to $D_{ \pm}$. Besides, by an assumption of type "the function $f$ satisfies $f_{ \pm} \in C^{p}\left(\bar{D}_{ \pm}\right)$" (or " $f \in C^{p}\left(\bar{D}_{+}\right) \cap C^{p}\left(\bar{D}_{-}\right)$") ) we will mean that the restriction of $f$ to $D_{+}$ (resp. $\left.D_{-}\right)$coincides on $D_{+}$(resp. $\left.D_{-}\right)$with a function $\tilde{f}_{+}$of class $C^{p}\left(\mathbb{R}^{d}\right)$ (resp. $\tilde{f}_{-}$). So that for any $x \in \Gamma$ we can give a sense for example to $f_{+}(x)$ : it is $\lim _{z \rightarrow x, z \in D_{+}} f(z)=\tilde{f}_{+}(x)$.

In the same time spirit we may note for $f \in C\left(\bar{D}_{+}\right) \cap C\left(\bar{D}_{-}\right)$and a point $y \in \Gamma$

$$
f(y \pm)=\lim _{z \rightarrow y, z \in D_{ \pm}} f(z)=f_{ \pm}(y)
$$

For $u \in C^{1}\left(\bar{D}_{+} ; \mathbb{R}\right) \cap C^{1}\left(\bar{D}_{-} ; \mathbb{R}\right)$ we denote $\nabla_{x} u=\left(\frac{\partial u}{\partial x_{1}}, \ldots, \frac{\partial u}{\partial x_{d}}\right)^{*}$ and, for a point $y \in \Gamma$

$$
\nabla_{x} u_{ \pm}(y)=\lim _{z \rightarrow y, z \in D_{ \pm}} \nabla_{x} u(z)
$$

For a vector field $G \in C^{1}\left(D ; \mathbb{R}^{d}\right)$ we denote by $\nabla \cdot G(x)$ its divergence at point $x \in D$, i.e. $\nabla \cdot G(x)=\sum_{i=1}^{d} \frac{\partial G_{i}}{\partial x_{i}}(x)$.

For $u \in C^{2}(D ; \mathbb{R})$ and $x \in D$ we denote $\mathbf{H}[u](x)$ the Hessian matrix of $u$ at point $x$.

Let $a(x)=\left(a_{i j}(x)\right)_{i, j \in\{1, \ldots, d\}}$ be a symmetric matrix valued and time homogeneous diffusion coefficient.

If $a_{i j} \in C^{1}(D ; \mathbb{R})$ for all $1 \leq i, j \leq d$ and $u \in C^{2}(D ; \mathbb{R})$ we denote

$$
\mathcal{L} u(x)=\nabla \cdot\left(a(x) \nabla_{x} u(x)\right), \quad \forall x \in D .
$$

In the whole paper the coefficients of the function matrix $a$ are always assumed to be measurable and bounded by a constant $\Lambda$.

We will also often make the following ellipticity assumption

Assumption 2.1. (E) : There exists $\lambda \in(0, \infty)$ such that

$$
\forall x \in \mathbb{R}^{d}, \quad \forall \xi \in \mathbb{R}^{d}, \quad \lambda|\xi|^{2} \leq \xi^{*} a(x) \xi
$$

Note that under $(\mathbf{E})$ we can assert that for any $x \in D$ we have

$$
a_{ \pm}(x)=P_{ \pm}^{*}(x) E_{ \pm}(x) P_{ \pm}(x)
$$

with $P_{ \pm}(x)$ some orthogonal matrices and $E_{ \pm}(x)$ some diagonal matrices with strictly positive eigenvalues.

Assume $\Gamma$ is $C^{2}$. For a point $x \in \Gamma$ we denote by $\nu(x) \in \mathbb{R}^{d}$ the unit normal to $\Gamma$ at point $x$, pointing to $D_{+}$. Assume the $a_{i j}$ 's satisfy $\left(a_{ \pm}\right)_{i j} \in C\left(\bar{D}_{ \pm}\right)$. 
We define then the co-normal vector fields $\gamma_{+}(x):=a_{+}(x) \nu(x)$ and $\gamma_{-}(x):=$ $-a_{-}(x) \nu(x)$, for $x \in \Gamma$.

Note that under $(\mathbf{E})$ it is clear that we have

$$
\forall x \in \Gamma, \quad\left\langle\gamma_{+}(x), \nu(x)\right\rangle \geq \lambda>0 \quad \text { and } \quad\left\langle\gamma_{-}(x), \nu(x)\right\rangle \leq-\lambda<0 .
$$

Note that the notation $\gamma_{ \pm}$for the trace operators follows the usual one ([38] for instance) and the notation $\gamma_{ \pm}$for the co-normal vectors follows the one of the paper [5]. But it will be dealt with the trace operator only in Section 3, and with co-normal vectors only in Sections 4 and 5 . So that these notations will cause no confusion.

In order to study the PDE aspects we will consider the unbounded operator $A: \mathcal{D}(A) \subset L^{2}\left(\mathbb{R}^{d}\right) \rightarrow L^{2}\left(\mathbb{R}^{d}\right)$ defined by

$$
\mathcal{D}(A)=\left\{u \in H^{1}\left(\mathbb{R}^{d}\right) \text { with } \sum_{i, j=1}^{d} D_{i}\left(a_{i j} D_{j} u\right) \in L^{2}\left(\mathbb{R}^{d}\right)\right\}
$$

and

$$
\forall u \in \mathcal{D}(A), \quad A u=\sum_{i, j=1}^{d} D_{i}\left(a_{i j} D_{j} u\right) .
$$

We also introduce the iterated domains defined recursively by

$$
\mathcal{D}\left(A^{k}\right)=\left\{v \in \mathcal{D}\left(A^{k-1}\right): \quad A v \in \mathcal{D}\left(A^{k-1}\right)\right\}, \quad k \geq 2 .
$$

These iterated domains will help us to establish the existence of a smooth solution to (1). First, using a variant of the Hille-Yosida theorem (Theorem VII-5 in [6]), we will prove that if the initial condition $u_{0}$ is taken in $\mathcal{D}\left(A^{k}\right), k \geq 2$ there is a solution $u$ to (1), which has classical smoothness in the time variable and weak smoothness in the space variable: for example this solution lives in $C\left([0, T] ; \mathcal{D}\left(A^{k}\right)\right)$ (we may speak of a semi-weak solution). Then we will prove that if $a$ has some additional smoothness in the domains $D_{ \pm}$, then a function $v$ in $\mathcal{D}\left(A^{k}\right)$ is of class $H^{2 k}$ if restricted to $D_{ \pm}$(but it is not necessarily of class $H^{2 k}$ on the whole space $\mathbb{R}^{d}$; see our Corollary 3.1). Using then Sobolev embedding arguments we will get classical smoothness of the semi-weak solution $u$ on each subdomain $D_{ \pm}$(see in particular the proof of Theorem 3.1).

\section{The parabolic transmission problem}

Let $0<T<\infty$ a finite time horizon. Let us consider the transmission parabolic problem 
$\left(\mathcal{P}_{\mathrm{T}}\right)\left\{\begin{aligned} \partial_{t} u(t, x)-\mathcal{L} u(t, x) & =0 & & \forall(t, x) \in(0, T] \times D \\ \left\langle a_{+} \nabla_{x} u_{+}(t, y)-a_{-} \nabla_{x} u_{-}(t, y), \nu(y)\right\rangle & =0 & & \forall(t, y) \in(0, T] \times \Gamma \\ u(t, y+) & =u(t, y-) & & \forall(t, y) \in[0, T] \times \Gamma \\ u(0, x) & =u_{0}(x) & & \forall x \in \mathbb{R}^{d} .\end{aligned}\right.$

We will say that $(t, x) \mapsto u(t, x)$ is classical solution to $\left(\mathcal{P}_{\mathrm{T}}\right)$ if it satisfies

$u \in C\left([0, T] ; C^{2}\left(\bar{D}_{+}\right) \cap C^{2}\left(\bar{D}_{-}\right)\right) \cap C^{1}\left([0, T] ; C\left(\bar{D}_{+}\right) \cap C\left(\bar{D}_{-}\right)\right) \cap C\left([0, T] ; C\left(\mathbb{R}^{d}\right)\right)$

and satisfies the following requisites. First, $u$ satisfies the first line of $\left(\mathcal{P}_{\mathrm{T}}\right)$, where the derivatives are understood in the classical sense. Second, for all $0<t \leq T$ the limits $\lim _{z \rightarrow y}, z \in D_{ \pm} \nabla_{x} u(t, z)$ satisfy the transmission condition $(\star)$ for all $y \in \Gamma$. Note that these limits exist thanks to (9). Third, $u$ is continuous accross $\Gamma$ (third line). Fourth, it satisfies the initial condition at the fourth line of $\left(\mathcal{P}_{\mathrm{T}}\right)$. The aim of this section is to prove the following result.

Theorem 3.1. Let $a=\left(a_{i j}\right)_{1 \leq i, j \leq d}$ satisfy $(\mathbf{E})$.

- Denote

$$
k_{0}=\left\{\begin{array}{l}
\left\lfloor\frac{d}{4}\right\rfloor+2 \text { if } d \text { is even } \\
\left\lfloor\frac{3}{2}+\frac{\lfloor d / 2\rfloor}{2}\right\rfloor+2 \text { if } d \text { is odd } .
\end{array}\right.
$$

Assume that the coefficients $a_{i j}$ satisfy $\left(a_{ \pm}\right)_{i j} \in C_{b}^{2 k_{0}-3}\left(\bar{D}_{ \pm}\right)$and $\Gamma$ is bounded and of class $C^{2 k_{0}-2}$. Then for $u_{0} \in \mathcal{D}\left(A^{k_{0}}\right)$ the parabolic transmission problem $\left(\mathcal{P}_{\mathrm{T}}\right)$ admits a classical solution.

- Furthermore, if $u_{0} \in \mathcal{D}\left(A^{k}\right)$ for $k \geq k_{0}$, the coefficients $a_{i j}$ satisfy $\left(a_{ \pm}\right)_{i j} \in$ $C_{b}^{2 k-1}\left(\bar{D}_{ \pm}\right)$and $\Gamma$ is bounded of class $C^{2 k}$, this classical solution $u$ is such that

$$
u \in C^{k-j}\left([0, T] ; C^{n(j)}\left(\bar{D}_{+}\right) \cap C^{n(j)}\left(\bar{D}_{-}\right)\right), \quad\lceil d / 4\rceil \leq j \leq k
$$

with $n(j)=\left\lfloor 2 j-\frac{d}{2}\right\rfloor$.

To prove Theorem 3.1 it requires to study in a first time the associated elliptic resolvent equation, in a weak sense. More precisely, for a source term $f \in L^{2}\left(\mathbb{R}^{d}\right)$ we will seek for a solution $u$ in $\mathcal{D}(A)$ of

$$
u-A u=f
$$

(see Proposition 3.4 below). 
Then the idea is to apply in $L^{2}\left(\mathbb{R}^{d}\right)$ a version of the Hille-Yosida theorem that states that for $u_{0} \in \mathcal{D}\left(A^{k}\right), k \geq 2$, there is a solution $u$ to $\frac{\mathrm{d} u}{\mathrm{~d} t}=A u, u(0)=u_{0}$, living in $C^{k-j}\left([0, T] ; \mathcal{D}\left(A^{j}\right)\right), 0 \leq j \leq k$ (see Proposition 3.6 below).

As we will have studied the weak smoothness of functions living in the $\mathcal{D}\left(A^{k}\right)$ 's (Proposition 3.5 and Corollary 3.1), we will be able to conclude that $u$ is in fact a classical solution by using Sobolev embedding arguments.

Remark 3.1. Note that in Theorem 3.1 we make no statement regarding the uniqueness of the classical solution of $\left(\mathcal{P}_{\mathrm{T}}\right)$. In order to prove uniqueness, a reasonable strategy could be to show that any classical solution is in fact a semiweak solution living in $C^{k-j}\left([0, T] ; \mathcal{D}\left(A^{j}\right)\right), 0 \leq j \leq k$. Such a semi-weak solution is known to be unique (see the forthcoming Proposition 3.6): more precisely two possible semi-weak solutions are equal at any time $t$ for almost every spatial point $x$; then using the continuity of classical solutions we would get the expected classical uniqueness result for the solution of problem $\left(\mathcal{P}_{\mathrm{T}}\right)$.

In our opinion it is possible to fulfill this purely PDE program, but we have chosen not to do so in this paper in order to avoid overburdening the exposition. Instead we adopt another strategy and prove that uniqueness in the classical sense holds as a consequence of the uniqueness in law of our stochastic numerical approximation scheme (that we build in the sequel) and its convergence towards any classical solution of $\left(\mathcal{P}_{\mathrm{T}}\right)$ (see the forthcoming Remarks 4.2 and 5.1 and the statement of Theorem 5.1).

Remark 3.2. 1) In the classical situation with smooth coefficients studied for instance in [15] Chap. 1 (or [31], Theorem 5.14), a unique classical solution to the parabolic PDE exists as soon as the $a_{i j}$ 's are bounded and Hölder continuous and satisfy $(\mathbf{E})$, and $u_{0}$ is continuous and satisfies some growth condition.

Here we ask additional smoothness on the coefficients $\left(a_{ \pm}\right)_{i j}$ 's inside the domains $D_{ \pm}$. Indeed, because of the discontinuity of a across $\Gamma$ we are led to use a different technique of proof: unlike the parametrix method in the classical case, this additional smoothness is required for the use of the Hille-Yosida theorem and the Sobolev embeddings.

Note that with this methodology of proof these additional assumptions would still be needed if our coefficients and the solution were smooth at the interface. Note also that with this approach the assumptions on the initial condition $u_{0}$ are understood in a weak sense (and are different).

2) Our result is also different from the one in [23] (Theorem 13.1; see also [24]). In this reference the authors study the classical smoothness of the parabolic transmission problem by studying first the smoothness of $\partial_{t} u$ (to that aim they differentiate with respect to time the initial equation). Then they study the smoothness with respect to the space variable by using results for the elliptic transmission problem, involving difference quotient techniques. But by doing so they get estimates on subdomains of the form $[\varepsilon, T] \times \bar{D}_{ \pm}$with $0<\varepsilon$. Here, we manage to study the global regularity of the classical solution of $\left(\mathcal{P}_{T}\right)$ in the whole domains $[0, T] \times \bar{D}_{ \pm}$. 
3.1. Study of the associated elliptic problem and of the domains $\mathcal{D}\left(A^{k}\right)$

In this subsection we establish the existence of a solution to (11) belonging to $\mathcal{D}(A)$ and study its smoothness properties, together with the ones of functions belonging to the iterated domains $\mathcal{D}\left(A^{k}\right)$, for $k \geq 1$.

We recall that the coefficients $a_{i j}$ are assumed to be bounded by $\Lambda$ so that we may define the following continuous bilinear and symmetric form, which will be used extensively in the sequel

$$
\mathcal{E}(u, v)=\sum_{i, j=1}^{d}\left\langle a_{i j} D_{j} u, D_{i} v\right\rangle_{L^{2}\left(\mathbb{R}^{d}\right)}, \quad \forall u, v \in H^{1}\left(\mathbb{R}^{d}\right) .
$$

Let $u \in \mathcal{D}(A)$. Using the definition of $A u$ as a distribution acting on $C_{c}^{\infty}\left(\mathbb{R}^{d} ; \mathbb{R}\right)$, and the density of $C_{c}^{\infty}\left(\mathbb{R}^{d} ; \mathbb{R}\right)$ in $H^{1}\left(\mathbb{R}^{d}\right)=H_{0}^{1}\left(\mathbb{R}^{d}\right)$, one can establish the following relation, linking $A$ and the form (12):

$$
\mathcal{E}(u, v)=\langle-A u, v\rangle_{L^{2}\left(\mathbb{R}^{d}\right)}, \quad \forall v \in H^{1}\left(\mathbb{R}^{d}\right) .
$$

\subsubsection{Some results on weak solutions of elliptic transmission PDEs}

Here we gather some preliminary results on weak solutions of elliptic transmission PDEs that rely mainly on [38] Chap. 4, pp. 141-145.

We recall that for $u \in L^{2}\left(\mathbb{R}^{d}\right)$, we denote $u_{+}$(resp. $u_{-}$) the restriction of $u$ to $D_{+}$(resp. $\left.D_{-}\right)$. It may happen that we use this notation for restricted distributions also.

We introduce the following notation for the jump across $\Gamma$ of $u \in L^{2}\left(\mathbb{R}^{d}\right)$, with $u_{+} \in H^{1}\left(D_{+}\right)$and $u_{-} \in H^{1}\left(D_{-}\right)$:

$$
[u]_{\Gamma}=\gamma_{+}\left(u_{+}\right)-\gamma_{-}(u-) .
$$

If $[u]_{\Gamma}=0$ we shall simply write $\gamma(u)=\gamma_{+}\left(u_{+}\right)=\gamma_{-}\left(u_{-}\right)$. We have the two following lemmas (the proof of the first one is straightforward).

Lemma 3.1. Let $v \in L^{2}\left(\mathbb{R}^{d}\right)$. Then, for any $1 \leq i \leq d$, the distribution $\left(D_{i} v\right)_{ \pm}$ is equal to $D_{i}\left(v_{ \pm}\right)$. As a consequence, if $v \in H^{1}\left(\mathbb{R}^{d}\right)$, then $v_{ \pm} \in H^{1}\left(D_{ \pm}\right)$.

Lemma 3.2 ([38], Exercise 4.5). Suppose $u \in L^{2}\left(\mathbb{R}^{d}\right)$ with $u_{ \pm} \in H^{1}\left(D_{ \pm}\right)$. Then $u \in H^{1}\left(\mathbb{R}^{d}\right)$ if and only if $[u]_{\Gamma}=0$ a.e. on $\Gamma$.

We shall consider restricted operators and bilinear forms in the following sense. We define $A_{+}: H^{1}\left(D_{+}\right) \rightarrow H^{-1}\left(D_{+}\right)$by

$$
\forall v \in H^{1}\left(D_{+}\right), \quad A_{+} v=\sum_{i, j=1}^{d} D_{i}\left(\left(a_{+}\right)_{i j} D_{j} v\right) .
$$

We define $A_{-}: H^{1}\left(D_{-}\right) \rightarrow H^{-1}\left(D_{-}\right)$in the same manner (note that we do not specify here any domain $\left.\mathcal{D}\left(A_{ \pm}\right)\right)$. Further, we define

$$
\mathcal{E}_{ \pm}(u, v)=\sum_{i, j=1}^{d} \int_{D_{ \pm}}\left(a_{ \pm}\right)_{i j} D_{j} u D_{i} v, \quad \forall u, v \in H^{1}\left(D_{ \pm}\right) .
$$


In the same fashion as for Equation (13), we have, for $u_{ \pm} \in H^{1}\left(D_{ \pm}\right)$with $A_{ \pm} u_{ \pm} \in L^{2}\left(D_{ \pm}\right)$

$$
\mathcal{E}_{ \pm}\left(u_{ \pm}, v\right)=\int_{D_{ \pm}}\left(-A_{ \pm} u_{ \pm}\right) v, \quad \forall v \in H_{0}^{1}\left(D_{ \pm}\right) .
$$

Imagine now that in (14) we wish to take the test function in $H^{1}\left(D_{ \pm}\right)$instead of $H_{0}^{1}\left(D_{ \pm}\right)$. There will still be a link between $A_{ \pm}$and $\mathcal{E}_{ \pm}$, but through Green type identities, involving co-normal derivatives and boundary integrals. We have the following result.

Proposition 3.1 (First Green identity, extended version; see [38] Theorem 4.4, point i)). Assume $\Gamma$ is bounded and $C^{2}$. Let $u \in L^{2}\left(\mathbb{R}^{d}\right)$ with $u_{+} \in H^{1}\left(D_{+}\right)$ and $u_{-} \in H^{1}\left(D_{-}\right)$. Assume $A_{+} u_{+} \in L^{2}(D+), A_{-} u_{-} \in L^{2}\left(D_{-}\right)$. Then there exist uniquely defined elements $\mathcal{B}_{\nu}^{ \pm} u \in H^{-\frac{1}{2}}(\Gamma)$ such that

$$
\mathcal{E}_{+}\left(u_{+}, v\right)=\int_{D_{+}}\left(-A_{+} u_{+}\right) v-\left(\mathcal{B}_{\nu}^{+} u, \gamma_{+}(v)\right)_{\Gamma}, \quad \forall v \in H^{1}\left(D_{+}\right)
$$

and

$$
\mathcal{E}_{-}\left(u_{-}, v\right)=\int_{D_{-}}\left(-A_{-} u_{-}\right) v+\left(\mathcal{B}_{\nu}^{-} u, \gamma_{-}(v)\right)_{\Gamma}, \quad \forall v \in H^{1}\left(D_{-}\right) .
$$

The elements $\mathcal{B}_{\nu}^{ \pm} u$ in Proposition 3.1 are the one-sided co-normal derivatives of $u$ on $\Gamma$.

To fix ideas, note that under the stronger assumptions that the $\left(a_{ \pm}\right)_{i j}$ 's are in $C_{b}^{1}\left(\bar{D}_{ \pm} ; \mathbb{R}\right)$, and $u_{ \pm} \in H^{2}\left(D_{ \pm}\right)$, we have

$$
\mathcal{B}_{\nu}^{ \pm} u=\nu^{*} \gamma_{ \pm}\left(a_{ \pm} \nabla u_{ \pm}\right)=\sum_{i=1}^{d} \sum_{j=1}^{d} \nu_{i} \gamma_{ \pm}\left(\left(a_{ \pm}\right)_{i j} D_{j} u_{ \pm}\right) \quad \text { on } \quad \Gamma
$$

(note that as the $\left(a_{ \pm}\right)_{i j} D_{j} u_{ \pm}$'s are in $H^{1}\left(D_{ \pm}\right)$the trace terms are correctly defined in the above expression). Thus one understands that the change of sign in front of the $(\cdot, \cdot)_{\Gamma}$ term between (15) and (16) is due to the fact that $-\nu$ is the outward normal to $D_{+}$and $\nu$ is the outward normal to $D_{-}$.

For details on the definition of $\mathcal{B}_{\nu}^{ \pm} u$ under the weaker assumptions of Proposition 3.1, see [38] pp. 116-117.

Finally we introduce a notation for the jumps across $\Gamma$ of the co-normal derivative of a function $u$ satisfying the assumptions of Proposition 3.1:

$$
\left[\mathcal{B}_{\nu} u\right]_{\Gamma}=\mathcal{B}_{\nu}^{+} u-\mathcal{B}_{\nu}^{-} u \in H^{-1 / 2}(\Gamma) .
$$

We have the following result.

Lemma 3.3 (Two-sided Green identity; inspired by [38] Lemma 4.19, Equation (4.33)). Assume $\Gamma$ is bounded and $C^{2}$. Let $u \in H^{1}\left(\mathbb{R}^{d}\right)$. Let $f_{+} \in L^{2}\left(D_{+}\right)$and $f_{-} \in L^{2}\left(D_{-}\right)$and assume

$$
u_{ \pm}-A_{ \pm} u_{ \pm}=f_{ \pm} \quad \text { on } D_{ \pm} .
$$


Set $f=f_{+}+f_{-}$, then

$$
\langle u, v\rangle_{L^{2}\left(\mathbb{R}^{d}\right)}+\mathcal{E}(u, v)=\langle f, v\rangle_{L^{2}\left(\mathbb{R}^{d}\right)}-\left(\left[\mathcal{B}_{\nu} u\right]_{\Gamma}, \gamma(v)\right)_{\Gamma}, \quad \forall v \in H^{1}\left(\mathbb{R}^{d}\right) .
$$

Remark 3.3. Note that in the above proposition $u_{ \pm} \in H^{1}\left(D_{ \pm}\right)$, thanks to Lemma 3.1. Equation (17) means that $\left\langle u_{ \pm}-A_{ \pm} u_{ \pm}, \varphi\right\rangle_{H^{-1}\left(D_{ \pm}\right), H_{0}^{1}\left(D_{ \pm}\right)}=\langle f, \varphi\rangle_{L^{2}\left(D_{ \pm}\right)}$, for all $\varphi \in C_{c}^{\infty}\left(D_{ \pm} ; \mathbb{R}\right)$. Therefore $A_{ \pm} u_{ \pm} \in L^{2}\left(D_{ \pm}\right)$and by Proposition 3.1 the element $\left[\mathcal{B}_{\nu} u\right]_{\Gamma}$ is well defined. Then same remark holds for the forthcoming Proposition 3.2.

Our notations being different from the ones in [38], we provide the short proof of Lemma 3.3 for the sake of clarity.

Proof. Taking into account Remark 3.3 we can use Proposition 3.1, and summing (15) and (16) one gets for any $v \in H^{1}\left(\mathbb{R}^{d}\right)$ (note that $\gamma_{+}\left(v_{+}\right)=\gamma_{-}\left(v_{-}\right)=$ $\gamma(v))$

$$
\langle u, v\rangle_{L^{2}\left(\mathbb{R}^{d}\right)}+\mathcal{E}_{+}\left(u_{+}, v_{+}\right)+\mathcal{E}_{-}\left(u_{-}, v_{-}\right)=\langle f, v\rangle_{L^{2}\left(\mathbb{R}^{d}\right)}-\left(\left[\mathcal{B}_{\nu} u\right]_{\Gamma}, \gamma(v)\right)_{\Gamma} .
$$

To complete the proof it suffices to notice that, thanks to Lemma 3.1, we have

$$
\begin{aligned}
\mathcal{E}_{+}\left(u_{+}, v_{+}\right)+\mathcal{E}_{-}\left(u_{-}, v_{-}\right) & =\sum_{i, j=1}^{d}\left\{\int_{D_{+}}\left(a_{+}\right)_{i j}\left(D_{j} u\right)_{+}\left(D_{i} v\right)_{+}\right. \\
& =\mathcal{E}(u, v) .
\end{aligned}
$$

We recall now results on the smoothness of weak solutions of elliptic transmission PDEs.

Proposition 3.2 ([38], Theorem 4.20). Let $G_{1}$ and $G_{2}$ be bounded open connected subsets of $\mathbb{R}^{d}$, such that $\overline{G_{1}} \subset G_{2}$ and $G_{1}$ intersects $\Gamma$, and put

$$
D_{ \pm}^{j}=G_{j} \cap D_{ \pm} \quad \text { and } \quad \Gamma_{j}=\Gamma \cap G_{j} \quad \text { for } j=1,2 .
$$

Assume that the set $G_{2}$ is constructed in such a way that there is a $C^{r+2}$ diffeomorphism between $\Gamma_{2}$ and a bounded portion of the hyperplane $x_{d}=0$.

Assume (E).

Let $r \in \mathbb{N}$. Assume that the coefficients $\left(a_{ \pm}\right)_{i j}$ belong to $C^{r+1}\left(\overline{D_{ \pm}^{2}} ; \mathbb{R}\right)$.

Let $f_{ \pm} \in L^{2}\left(D_{ \pm}\right)$with $f_{ \pm} \in H^{r}\left(D_{ \pm}^{2}\right)$. Let $u \in L^{2}\left(\mathbb{R}^{d}\right)$ with $u \in H^{1}\left(G_{2}\right)$ satisfying

$$
u_{ \pm}-A_{ \pm} u_{ \pm}=f_{ \pm} \quad \text { on } D_{ \pm}^{2}
$$

and $\left[\mathcal{B}_{\nu} u\right]_{\Gamma} \in H^{\frac{1}{2}+r}\left(\Gamma_{2}\right)$. Then $u_{ \pm} \in H^{2+r}\left(D_{ \pm}^{1}\right)$. 
Proposition 3.3 ([18], Theorem 8.10). Assume (E).

Let $r \in \mathbb{N}$. Assume that the coefficients $\left(a_{ \pm}\right)_{i j}$ belong to $C_{b}^{r+1}\left(\bar{D}_{ \pm} ; \mathbb{R}\right)$. Assume $\Gamma$ is bounded.

Let $f_{ \pm} \in H^{r}\left(D_{ \pm}\right)$. Let $u \in H^{1}\left(\mathbb{R}^{d}\right)$ satisfying

$$
u_{ \pm}-A_{ \pm} u_{ \pm}=f_{ \pm} \quad \text { on } D_{ \pm} .
$$

Let $D_{ \pm}^{\prime} \subset D_{ \pm}$open subsets with $\overline{D_{ \pm}^{\prime}} \subset D_{ \pm}$and denote $d_{ \pm}^{\prime}=\operatorname{dist}\left(D_{ \pm}^{\prime}, \Gamma\right)$.

We have that $u_{ \pm} \in H^{r+2}\left(D_{ \pm}^{\prime}\right)$, with

$$
\left\|u_{ \pm}\right\|_{H^{r+2}\left(D_{ \pm}^{\prime}\right)} \leq C_{ \pm}\left(\left\|u_{ \pm}\right\|_{H^{1}\left(D_{ \pm}\right)}+\|f\|_{H^{r}\left(D_{ \pm}\right)}\right),
$$

where the constant $C_{ \pm}$depends on $d, \lambda, d_{ \pm}^{\prime}$ and

$$
\max _{1 \leq i, j \leq d} \max _{|\alpha| \leq r+1} \sup _{x \in D_{ \pm}}\left|\partial^{\alpha}\left(a_{ \pm}\right)_{i j}(x)\right| .
$$

Proof. In [18] this result is asserted with the assumption that $\overline{D_{ \pm}^{\prime}} \subset D_{ \pm}$, with $\overline{D_{ \pm}^{\prime}}$ compact. So that for the interior (bounded) domain $D_{+}$the result is immediate. On the unbounded domain $D_{-}$we claim that the same result holds for non compact $\overline{D_{-}^{\prime}}$, as in fact only the distance $d_{-}^{\prime}=\operatorname{dist}\left(D_{-}^{\prime}, \Gamma\right)$ plays a role in the proof.

Thus, covering $\Gamma$ with open balls in order to use the local result of Proposition 3.2, and combining with the global result of Proposition 3.3, it is possible to show the following theorem, that will be used extensively in the sequel.

Theorem 3.2. Assume (E).

Let $r \in \mathbb{N}$. Assume that the coefficients $\left(a_{ \pm}\right)_{i j}$ belong to $C_{b}^{r+1}\left(\bar{D}_{ \pm} ; \mathbb{R}\right)$. Assume $\Gamma$ is bounded and of class $C^{r+2}$.

Let $f_{ \pm} \in H^{r}\left(D_{ \pm}\right)$. Let $u \in H^{1}\left(\mathbb{R}^{d}\right)$ satisfying

$$
u_{ \pm}-A_{ \pm} u_{ \pm}=f_{ \pm} \quad \text { on } D_{ \pm}
$$

and $\left[\mathcal{B}_{\nu} u\right]_{\Gamma} \in H^{\frac{1}{2}+r}(\Gamma)$. Then $u_{ \pm} \in H^{2+r}\left(D_{ \pm}\right)$.

3.1.2. Existence of a weak solution to the resolvent equation and immediate properties of functions in $\mathcal{D}\left(A^{k}\right), k \geq 1$

We have the next result.

Proposition 3.4. Assume (E). Let $f \in L^{2}\left(\mathbb{R}^{d}\right)$. Then (11) has a unique solution in $\mathcal{D}(A)$.

Proof. Let us note that the symmetric bilinear form on $H^{1}\left(\mathbb{R}^{d}\right)$

$$
(u, v) \mapsto\langle u, v\rangle_{L^{2}\left(\mathbb{R}^{d}\right)}+\mathcal{E}(u, v)
$$

is continuous and, thanks to Assumption (E), coercive. Thus the Lax-Milgram theorem ([6] Corollary V.8) immediately asserts the existence of a unique $u \in$ $H^{1}\left(\mathbb{R}^{d}\right)$ such that

$$
\forall v \in H^{1}\left(\mathbb{R}^{d}\right), \quad\langle u, v\rangle_{L^{2}\left(\mathbb{R}^{d}\right)}+\mathcal{E}(u, v)=\langle f, v\rangle_{L^{2}\left(\mathbb{R}^{d}\right)} .
$$


In other words we have for any $\varphi \in C_{c}^{\infty}\left(\mathbb{R}^{d} ; \mathbb{R}\right)$,

$$
\mathcal{E}(u, \varphi)=-\left\langle\sum_{i, j=1}^{d} D_{i}\left(a_{i j} D_{j} u\right), \varphi\right\rangle_{H^{-1}\left(\mathbb{R}^{d}\right), H^{1}\left(\mathbb{R}^{d}\right)}=\langle(f-u), \varphi\rangle_{L^{2}\left(\mathbb{R}^{d}\right)} .
$$

Hence the distribution $\sum_{i, j=1}^{d} D_{i}\left(a_{i j} D_{j} u\right)$ belongs to $L^{2}\left(\mathbb{R}^{d}\right)$, and thus $u \in$ $\mathcal{D}(A)$. Finally, from the above relations we deduce

$$
\forall v \in H^{1}\left(\mathbb{R}^{d}\right), \quad\langle u-A u, v\rangle_{L^{2}\left(\mathbb{R}^{d}\right)}=\langle f, v\rangle_{L^{2}\left(\mathbb{R}^{d}\right)},
$$

which implies (11).

The proposition below gives properties of functions belonging to $\mathcal{D}(A)$. It indicates that the solution $u \in \mathcal{D}(A)$ of (11) encountered in Proposition 3.4 satisfies a continuity property and a transmission condition in a weak sense at the interface.

Proposition 3.5. Let $u \in \mathcal{D}(A)$. Then $[u]_{\Gamma}=\left[\mathcal{B}_{\nu} u\right]_{\Gamma}=0$ a.e. on $\Gamma$.

Proof. Let $u \in \mathcal{D}(A)$. As $u \in H^{1}\left(\mathbb{R}^{d}\right)$ one gets by Lemma 3.2 that $[u]_{\Gamma}=0$ a.e. on $\Gamma$. Set now $f=u-A u \in L^{2}\left(\mathbb{R}^{d}\right)$. According to Equation (13) we have

$$
\forall v \in H^{1}\left(\mathbb{R}^{d}\right), \quad\langle u, v\rangle_{L^{2}\left(\mathbb{R}^{d}\right)}+\mathcal{E}(u, v)=\langle f, v\rangle_{L^{2}\left(\mathbb{R}^{d}\right)},
$$

and this in true in particular for any $v \in C_{c}^{\infty}\left(D_{+} ; \mathbb{R}\right)$. But using Lemma 3.1 one has for any $v \in C_{c}^{\infty}\left(D_{+} ; \mathbb{R}\right)$, that

$\mathcal{E}(u, v)=\sum_{i, j=1}^{d} \int_{D_{+}}\left(a_{+}\right)_{i j}\left(D_{j} u\right)_{+} D_{i} v=\sum_{i, j=1}^{d} \int_{D_{+}}\left(a_{+}\right)_{i j}\left(D_{j} u_{+}\right) D_{i} v=\mathcal{E}_{+}\left(u_{+}, v\right)$.

Using now (14) we see that $\left\langle u_{+}-A_{+} u_{+}, v\right\rangle_{L^{2}\left(D_{+}\right)}=\left\langle f_{+}, v\right\rangle_{L^{2}\left(D_{+}\right)}$for any $v \in C_{c}^{\infty}\left(D_{+} ; \mathbb{R}\right)$. Proceeding in the same manner on $D_{-}$we finally see that $u_{ \pm}-A_{ \pm} u_{ \pm}=f_{ \pm}$on $D_{ \pm}$.

Note that by construction $f=f_{+}+f_{-}$. Using now Lemma 3.3, and comparing (18) and (19), one gets $\left(\left[\mathcal{B}_{\nu} u\right]_{\Gamma}, \gamma(v)\right)_{\Gamma}=0$ for any $v \in H^{1}\left(\mathbb{R}^{d}\right)$. Using the fact that the trace operator is surjective, this implies that $\left(\left[\mathcal{B}_{\nu} u\right]_{\Gamma}, w\right)_{\Gamma}=0$ for any $w \in H^{1 / 2}(\Gamma)$, which completes the proof.

Thanks to Theorem 3.2 we can get as a corollary the following result concerning the iterated domains $\mathcal{D}\left(A^{k}\right), k \in \mathbb{N}^{*}$.

Corollary 3.1. Assume $(\mathbf{E})$. Let $k \in \mathbb{N}^{*}$ and $u \in \mathcal{D}\left(A^{k}\right)$. Assume that the coefficients $\left(a_{ \pm}\right)_{i j} \in C_{b}^{2 k-1}\left(D_{ \pm}\right)$and that $\Gamma$ is bounded and of class $C^{2 k}$. Then $u_{ \pm} \in H^{2 k}\left(D_{ \pm}\right)$.

Proof. The proof proceeds by induction on $k$.

Let $u \in \mathcal{D}(A)($ case $k=1)$. We have $\left[\mathcal{B}_{\nu} u\right]_{\Gamma}=0$, according to Proposition 3.5. Thus in particular $\left[\mathcal{B}_{\nu} u\right]_{\Gamma} \in H^{\frac{1}{2}}(\Gamma)$. As in the proof of Proposition 3.5 
we set $f=u-A u$ and notice that we have $u_{ \pm}-A_{ \pm} u_{ \pm}=f_{ \pm}$on $D_{ \pm}$, with $f_{ \pm} \in L^{2}\left(D_{ \pm}\right)$.

Using Theorem 3.2 - remember that $u$ is in $H^{1}\left(\mathbb{R}^{d}\right),\left(a_{ \pm}\right)_{i j} \in C_{b}^{1}\left(\bar{D}_{ \pm} ; \mathbb{R}\right)$ and $\Gamma$ is bounded of class $C^{2}$ - we get that $u_{ \pm} \in H^{2}\left(D_{ \pm}\right)$.

Suppose now that the result is true at rank $k-1$ we prove its validity at $\operatorname{rank} k(k \geq 2)$. Let $u \in \mathcal{D}\left(A^{k}\right)$. As $u \in \mathcal{D}(A)$ we have $\left[\mathcal{B}_{\nu} u\right]_{\Gamma}=0 \in H^{2 k-\frac{3}{2}}(\Gamma)$. As $A u \in \mathcal{D}\left(A^{k-1}\right)$ the quantity $u-A u=: f$ satisfies $f_{ \pm} \in H^{2 k-2}\left(D_{ \pm}\right)$, using the induction hypothesis. But as we have $u_{ \pm}-A_{ \pm} u_{ \pm}=f_{ \pm}$on $D_{ \pm}$, one may use again the smoothness of $\left(a_{ \pm}\right)_{i j}$ and $\Gamma$ and Theorem 3.2 in order to conclude that $u_{ \pm} \in H^{2 k}\left(D_{ \pm}\right)$.

\subsection{The solution of the parabolic problem $\left(\mathcal{P}_{\mathrm{T}}\right)$}

\subsubsection{Application of the Hille-Yosida theorem}

We now use the Hille-Yosida theorem ([6] Theorems VII.4 and VII.5) in order to prove the following proposition. Note that in Equation (20) below, the time derivative is understood in the strong sense, while the space derivatives are understood in the weak sense. Besides, by convention $\mathcal{D}\left(A^{0}\right)=L^{2}\left(\mathbb{R}^{d}\right)$.

Proposition 3.6. Assume $(\mathbf{E})$. Let $u_{0} \in \mathcal{D}(A)$. Then there exists a unique function

$$
u \in C^{1}\left([0, T] ; L^{2}\left(\mathbb{R}^{d}\right)\right) \cap C([0, T] ; \mathcal{D}(A))
$$

satisfying

$$
\frac{\mathrm{d} u}{\mathrm{~d} t}=A u, \quad u(0)=u_{0} .
$$

Furthermore, let $u_{0} \in \mathcal{D}\left(A^{k}\right), k \geq 2$. Then,

$$
u \in C^{k-j}\left([0, T] ; \mathcal{D}\left(A^{j}\right)\right), \quad 0 \leq j \leq k .
$$

Proof. According to [6] it suffices to check that $(-A, \mathcal{D}(A))$ is maximal monotone. But thanks to Assumption (E) we immediately see that $\langle-A v, v\rangle_{L^{2}\left(\mathbb{R}^{d}\right)}=$ $\mathcal{E}(v, v) \geq 0$, for any $v \in \mathcal{D}(A)$, and thanks to Proposition 3.4 we have that for any $f \in L^{2}\left(\mathbb{R}^{d}\right)$ there exists $u \in \mathcal{D}(A)$ solving (11).

Using now Proposition 3.5, Corollary 3.1 and Proposition 3.6 together with some Sobolev embedding theorems, we show Theorem 3.1.

\subsubsection{Proof of Theorem 3.1}

Proof. Assume $d$ is even. Apply the result of Proposition 3.6 with $k=k_{0}=$ $\left\lfloor\frac{d}{4}\right\rfloor+2$ and consider $u$ solution of (20). We have that

$$
u \in C^{1}\left([0, T] ; \mathcal{D}\left(A^{k_{0}-1}\right)\right)
$$

with $k_{0}-1=\left\lfloor\frac{d}{4}\right\rfloor+1$. Using the result of Corollary 3.1 and combining Corollary IX.13 p. 168 with Theorem IX.7 p. 157 in [6], we see that for any $t \in[0, T]$

$$
u_{ \pm}(t, .) \in H^{4+2\left\lfloor\frac{d}{4}\right\rfloor}\left(D_{ \pm}\right) \subset H^{2+\frac{d}{2}}\left(D_{ \pm}\right) \hookrightarrow C^{2}\left(\bar{D}_{ \pm}\right) .
$$


Assume now that $d$ is odd. Apply the result of Proposition 3.6 with $k=$ $k_{0}=\left\lfloor\frac{3}{2}+\frac{\lfloor d / 2\rfloor}{2}\right\rfloor+2$ and consider $u$ solution of (20). We have that

$$
u \in C^{1}\left([0, T] ; \mathcal{D}\left(A^{k_{0}-1}\right)\right)
$$

with $k_{0}-1=\left\lfloor\frac{3}{2}+\frac{\lfloor d / 2\rfloor}{2}\right\rfloor+1$. Using the result of Corollary 3.1 and combining Corollary IX.13 p. 168 with Theorem IX.7 p. 157 in [6], we see that for any $t \in[0, T]$

$$
u_{ \pm}(t, .) \in H^{2+2\left\lfloor\frac{3}{2}+\left\lfloor\frac{\lfloor d / 2\rfloor}{2}\right\rfloor\right.}\left(D_{ \pm}\right) \hookrightarrow C^{2}\left(\bar{D}_{ \pm}\right)
$$

since

$$
\left\lfloor 2+2\left\lfloor\frac{3}{2}+\frac{\lfloor d / 2\rfloor}{2}\right\rfloor-\frac{d}{2}\right\rfloor \geq\left\lfloor 2+2\left(\frac{1}{2}+\frac{\lfloor d / 2\rfloor}{2}\right)-\frac{d}{2}\right\rfloor \geq\left\lfloor 3+\left\lfloor\frac{d}{2}\right\rfloor-\frac{d}{2}\right\rfloor \geq 2 .
$$

Let us now show that $u$ solution of (20) (for the corresponding $k_{0}$ ) is a classical solution of $\left(\mathcal{P}_{\mathrm{T}}\right)$.

First, it is clear that $\mathcal{L} u$ coincides with $A u$ on any bounded part of $D_{ \pm}$ (the derivatives in the distributional sense coincide with the classical derivatives thanks to the established smoothness of $u)$. This shows the first line of $\left(\mathcal{P}_{\mathrm{T}}\right)$.

Second, as for any $t \in[0, T]$ the function $u(t,$.$) belongs to \mathcal{D}(A)$, we have using the result of Proposition 3.5 that

$$
[u(t, .)]_{\Gamma}=0 \text { a.e. on } \Gamma ; \quad\left[\mathcal{B}_{\nu} u(t, .)\right]_{\Gamma}=0 \text { a.e. on } \Gamma \text {. }
$$

Note that $u(t,.) \in \mathcal{D}(A)$ implies that $u_{ \pm}(t,$.$) are in H^{2}\left(D_{ \pm}\right)$. So that the second part of (23) reads

$$
\nu^{*}\left(\gamma_{+}\left(a_{+} \nabla u_{+}(t, .)\right)-\gamma_{-}\left(a_{-} \nabla u_{-}(t, .)\right)\right)=0 \quad \text { a.e. }
$$

But as $\left(a_{ \pm} \nabla u_{ \pm}\right) \in C^{1}\left(\bar{D}_{ \pm} ; \mathbb{R}^{d}\right)$, we get

$$
\left\langle\left(a_{+} \nabla_{x} u_{+}(t, .)\right)(y)-\left(a_{-} \nabla_{x} u_{-}(t, .)\right)(y), \nu(y)\right\rangle=0
$$

for almost every $y \in \Gamma$, and consequently for every $y \in \Gamma$ by continuity. The same argument applies to the first part of (23) and the second and third lines of $\left(\mathcal{P}_{\mathrm{T}}\right)$ are satisfied. Note that the constructed solution satisfies $u(t,.) \in C\left(\mathbb{R}^{d}\right)$ for any time $t \in[0, T]$.

Now let $k \geq k_{0}$. For $\left\lceil\frac{d}{4}\right\rceil \leq j \leq k$, we have $2 j-\frac{d}{2}>0$. Thus, for $v \in \mathcal{D}\left(A^{j}\right)$ we have from Corollary 3.1,

$$
v_{ \pm} \in H^{2 j}\left(D_{ \pm}\right) \hookrightarrow C^{n(j)}\left(\bar{D}_{ \pm}\right)
$$

with $n(j)=\left\lfloor 2 j-\frac{d}{2}\right\rfloor$. Using again the result of Proposition 3.6, we get the announced result. 


\subsection{Conclusion and consequences: boundedness of the partial derivatives}

Going a bit further in the analysis, and using additional Sobolev embedding arguments, we can state the following result.

Proposition 3.7. Assume (E). Let $p, q \in \mathbb{N}$ with $p+\lfloor q / 2\rfloor \geq 2$. Let $m=$ $\left\lceil\frac{q}{2}+\frac{d}{4}\right\rceil, m^{\prime}=m+1$ and $k=m^{\prime}+p$. Assume that the coefficients $a_{i j}$ satisfy $\left(a_{ \pm}\right)_{i j} \in C_{b}^{2 m^{\prime}-1}\left(\bar{D}_{ \pm}\right)$, that $\Gamma$ is bounded and of class $C^{2 m^{\prime}}$, and that $u_{0} \in$ $\mathcal{D}\left(A^{k}\right)$.

Then the classical solution $u(t, x)$ of $\left(\mathcal{P}_{\mathrm{T}}\right)$ constructed in Theorem 3.1 satisfies

$$
u \in C^{p}\left([0, T] ; C_{b}^{q}\left(\bar{D}_{+}\right) \cap C_{b}^{q}\left(\bar{D}_{-}\right)\right) .
$$

Proof. First, notice that it is easy to check that $k$ is greater than the $k_{0}$ defined in Theorem 3.1, so that it makes sense speaking of the classical solution of $\left(\mathcal{P}_{\mathrm{T}}\right)$, for $u_{0} \in \mathcal{D}\left(A^{k}\right)$.

This solution is constructed in the same way as in Theorem 3.1, in particular by the mean of Proposition 3.6. So that one can assert that

$$
u \in C^{p}\left([0, T] ; \mathcal{D}\left(A^{m^{\prime}}\right)\right) .
$$

It remains to check that if $v \in \mathcal{D}\left(A^{m^{\prime}}\right)$, then $v_{ \pm} \in C_{b}^{q}\left(\bar{D}_{ \pm}\right)$. First, note that $m \geq\left\lceil\frac{d}{4}\right\rceil$, and that one may easily check

$$
\left\lfloor 2 m-\frac{d}{2}\right\rfloor \geq q
$$

(using in particular $\lceil 2 a\rceil \leq 2\lceil a\rceil)$. So that if $v \in \mathcal{D}\left(A^{m^{\prime}}\right) \subset \mathcal{D}\left(A^{m}\right)$, we have, as for the second part of Theorem 3.1,

$$
v_{ \pm} \in H^{2 m}\left(D_{ \pm}\right) \hookrightarrow C^{\left\lfloor 2 m-\frac{d}{2}\right\rfloor}\left(\bar{D}_{ \pm}\right) \subset C^{q}\left(\bar{D}_{ \pm}\right) .
$$

We claim that for any multi-index $\alpha,|\alpha| \leq q$, the partial derivatives $\partial^{\alpha} v_{ \pm}$are bounded. Indeed, using again Corollary 3.1 , we get

$$
v_{ \pm} \in H^{2 m^{\prime}}\left(D_{ \pm}\right)
$$

so that for $\alpha,|\alpha| \leq q$,

$$
\partial^{\alpha} v_{ \pm} \in H^{2\left\lceil\frac{q}{2}+\frac{d}{4}\right\rceil-q+2}\left(D_{ \pm}\right) \subset H^{\frac{d}{2}+2}\left(D_{ \pm}\right) \hookrightarrow L^{\infty}\left(D_{ \pm}\right) .
$$

Here we have used the fact $\frac{1}{2}-\frac{1}{2}-\frac{2}{d}<0$, so that one can use the third embedding result of Corollary IX.13 in [6] (and again Theorem IX.7 for the projection argument). The result is proved.

From the above proposition we get the following control on the partial derivatives of the solution to $\left(\mathcal{P}_{\mathrm{T}}\right)$. 
Corollary 3.2. In the context of Proposition 3.7 we have

$$
\sup _{t \in[0, T]} \sup _{x \in \bar{D}_{ \pm}}\left|\partial_{t}^{j} \partial^{\alpha} u_{ \pm}(t, x)\right|<\infty
$$

for any $j \leq p$ and any multi-index $\alpha$, with $|\alpha| \leq q$.

Proof. By Proposition 3.7 any of the considered partial derivatives of $u_{ \pm}$belongs to the space

$$
C\left([0, T] ; C_{b}\left(\bar{D}_{ \pm}\right)\right)
$$

Let for example $v \in C\left([0, T] ; C_{b}\left(\bar{D}_{+}\right)\right)$. We prove the continuity of the map $t \mapsto \sup _{x \in \bar{D}_{+}}|v(t, x)|, t \in[0, T]$. Let $t_{0} \in[0, T]$. Using the reverse triangle inequality we get for any $t \neq t_{0}$,

$$
\left|\sup _{x \in \bar{D}_{+}}\right| v(t, x)\left|-\sup _{x \in \bar{D}_{+}}\right| v\left(t_{0}, x\right)|| \leq \sup _{x \in \bar{D}_{+}}\left|v(t, x)-v\left(t_{0}, x\right)\right|,
$$

and we get the continuity at $t_{0}$, as $v$ is continuous from $[0, T]$ to $C_{b}\left(\bar{D}_{+}\right)$ (equipped with the supreme norm). Thus the desired continuity is proved, and from this we can assert that

$$
\sup _{t \in[0, T]} \sup _{x \in \bar{D}_{+}}|v(t, x)|=\sup _{x \in \bar{D}_{+}}\left|v\left(t^{*}, x\right)\right|
$$

for some $t^{*} \in[0, T]$. As $v\left(t^{*}, \cdot\right) \in C_{b}\left(\bar{D}_{+}\right)$we have that

$$
\sup _{t \in[0, T]} \sup _{x \in \bar{D}_{+}}|v(t, x)|<\infty .
$$

The result is proved.

In the analysis of the convergence of our Euler scheme, we will use the above corollary with $p$ up to 2 and $q$ up to 4 .

\section{Euler scheme}

4.1. Recalls on the projection and the distance to the transmission boundary and further notations and premiminaries

In this subsection we adopt the notations from [5]. We have the following set of geometric results.

Proposition 4.1 ([5], Proposition 1; see also [19]). Assume $\Gamma$ is bounded and of class $C^{5}$. Assume $(\mathbf{E})$. Assume that the coefficients $a_{i j}$ satisfy $\left(a_{ \pm}\right)_{i j} \in$ $C_{b}^{4}\left(\bar{D}_{ \pm}\right)$

There is constant $R>0$ such that:

1. (a) for any $x \in V_{\Gamma}^{-}(R)$, there are unique $s=\pi_{\Gamma}^{\gamma_{+}}(x) \in \Gamma$ and $F^{\gamma_{+}}(x) \leq 0$ such that:

$$
x=\pi_{\Gamma}^{\gamma_{+}}(x)+F^{\gamma_{+}}(x) \gamma_{+}\left(\pi_{\Gamma}^{\gamma_{+}}(x)\right)
$$


(b) for any $x \in V_{\Gamma}^{+}(R)$, there are unique $s=\pi_{\Gamma}^{\gamma_{-}}(x) \in \Gamma$ and $F^{\gamma-}(x) \leq 0$ such that:

$$
x=\pi_{\Gamma}^{\gamma-}(x)+F^{\gamma_{-}}(x) \gamma_{-}\left(\pi_{\Gamma}^{\gamma_{-}}(x)\right) ;
$$

2. (a) the function $x \mapsto \pi_{\Gamma}^{\gamma_{+}}(x)$ is called the projection of $x$ on $\Gamma$ parallel to $\gamma_{+}$: this is a $C^{4}$ function on $V_{\Gamma}^{-}(R)$;

(b) the function $x \mapsto \pi_{\Gamma}^{\gamma-}(x)$ is called the projection of $x$ on $\Gamma$ parallel to $\gamma_{-}$: this is a $C^{4}$ function on $V_{\Gamma}^{+}(R)$;

3. Let us set $\tilde{F}^{\gamma_{ \pm}}(x)=F^{\gamma_{ \pm}}(x)\left|\gamma_{ \pm}\left(\pi_{\Gamma}^{\gamma_{ \pm}}(x)\right)\right|$ the normalized version of $F^{\gamma_{ \pm}}$ corresponding to the unit vector field $\tilde{\gamma}_{ \pm}: x \mapsto \frac{\gamma_{ \pm}(x)}{\left|\gamma_{ \pm}(x)\right|}$.

(a) the functions $x \mapsto \tilde{F}^{\gamma_{ \pm}}(x)$ are called the algebraic distance of $x$ to $\Gamma$ parallel to $\gamma_{ \pm}\left(\right.$to $\left.\tilde{\gamma}_{ \pm}\right)$: these are $C^{4}$ functions on $V_{\Gamma}^{\mp}(R)$. One has $F^{\gamma_{+}}, \tilde{F}^{\gamma_{+}} \leq 0$ on $V_{\Gamma}^{-}(R)$ and $F^{\gamma_{-}}, \tilde{F}^{\gamma_{-}} \leq 0$ on $V_{\Gamma}^{+}(R)$.

(b) It is possible to extend $F^{\gamma_{+}}, \tilde{F}^{\gamma_{+}}$and $F^{\gamma_{-}}, \tilde{F}^{\gamma_{-}}$to $C_{b}^{4}\left(\mathbb{R}^{d}, \mathbb{R}\right)$ functions, with the conditions $F^{\gamma_{ \pm}}, \tilde{F}^{\gamma_{ \pm}}>0$ on $D_{ \pm}$and $F^{\gamma_{ \pm}}, \tilde{F}^{\gamma_{ \pm}}<0$ on $D_{\mp}$.

4. The above extensions for $\tilde{F}^{\gamma_{ \pm}}$and $F^{\nu}$ can be performed in a way such that the functions $\tilde{F}^{\gamma_{ \pm}}$and $F^{\nu}$ are equivalent in the sense that for all $x \in \mathbb{R}^{d}$,

$$
\frac{1}{c_{1}} d(x, \Gamma)=\frac{1}{c_{1}}\left|F^{\nu}(x)\right| \leq\left|\tilde{F}^{\gamma_{ \pm}}(x)\right| \leq c_{1}\left|F^{\nu}(x)\right|=c_{1} d(x, \Gamma)
$$

for some constant $c_{1}>1$.

5. For $x \in \Gamma$,

$$
\nabla \tilde{F}^{\gamma_{ \pm}}(x)=\frac{\nu^{*}}{\left\langle\nu, \tilde{\gamma}_{ \pm}\right\rangle}(x) .
$$

Remark 4.1. Under the assumptions of Proposition 4.1 we have that the vector fields $\gamma_{ \pm}(x), x \in \Gamma$, are of class $C^{4}$, and we have (8). Thus we are indeed under the assumptions of Proposition 1 in [5].

We sometimes use the notation $\nu(x)$ or $\gamma_{ \pm}(x)$ even if $x \notin \Gamma$. For $x \in V_{\Gamma}^{ \pm}(R)$, we set $\nu(x)=\nu\left(\pi_{\Gamma}^{\gamma_{ \pm}}(x)\right)$ and $\gamma_{ \pm}(x)=\gamma_{ \pm}\left(\pi_{\Gamma}^{\gamma_{ \pm}}(x)\right)$ and for $x \notin V_{\Gamma}^{ \pm}(R)$, arbitrary values are given.

Note that if $u$ is a classical solution to the transmission parabolic problem $\left(\mathcal{P}_{\mathrm{T}}\right)$ defined in Section 3, the transmission condition $(\star)$ can be expressed as

$$
\left\langle\gamma_{+}(y), \nabla_{x} u_{+}(t, y)\right\rangle=-\left\langle\gamma_{-}(y), \nabla_{x} u_{-}(t, y)\right\rangle, \quad \forall(t, y) \in(0, T] \times \Gamma .
$$

This in fact will be the crux of our approach (see Subsubsection 5.5.2).

In the sequel, we will need the following result.

Proposition 4.2. Assume $\Gamma$ is bounded and of class $C^{5}$. Assume $(\mathbf{E})$. Assume that the coefficients $a_{i j}$ satisfy $\left(a_{ \pm}\right)_{i j} \in C_{b}^{4}\left(\bar{D}_{ \pm}\right)$.

Let $\hat{x} \in V_{\Gamma}^{\mp}(R)$ and $\bar{x} \in V_{\Gamma}^{\mp}(R)$ be linked by the following relation :

$$
\bar{x}=\pi_{\Gamma}^{\gamma_{ \pm}}(\hat{x})-F^{\gamma_{ \pm}}(\hat{x}) \gamma_{\mp}\left(\pi_{\Gamma}^{\gamma_{ \pm}}(\hat{x})\right) .
$$


Then, there exists $c_{2}>1$ such that

$$
\frac{1}{c_{2}} d(\bar{x}, \Gamma) \leq d(\hat{x}, \Gamma) \leq c_{2} d(\bar{x}, \Gamma) .
$$

Proof. Without loss of generality, assume for example that $\bar{x} \in V_{\Gamma}^{-}(R)$ and $\hat{x} \in V_{\Gamma}^{-}(R)$ are related by (29). Then we have

$$
\bar{x}-\pi_{\Gamma}^{\gamma_{+}}(\hat{x})=-F^{\gamma_{+}}(\hat{x}) \gamma_{-}\left(\pi_{\Gamma}^{\gamma_{+}}(\hat{x})\right) .
$$

and by uniqueness of the projection $\pi_{\Gamma}^{-\gamma_{-}}(\bar{x})$, we see that $\pi_{\Gamma}^{\gamma+}(\hat{x})=\pi_{\Gamma}^{-\gamma_{-}}(\bar{x})$ (note that $\left.F^{-\gamma_{-}}(\bar{x})=F^{\gamma_{+}}(\hat{x})\right)$.

We deduce that

$$
\begin{array}{r}
\frac{1}{c_{1}} d(\bar{x}, \Gamma) \leq\left|\tilde{F}^{-\gamma_{-}}(\bar{x})\right|=\left|F^{-\gamma_{-}}(\bar{x})\right| \times\left|\gamma_{-}\left(\pi_{\Gamma}^{-\gamma_{-}}(\bar{x})\right)\right|=\left|\bar{x}-\pi_{\Gamma}^{\gamma_{+}}(\hat{x})\right| \\
=\left|\bar{x}-\pi_{\Gamma}^{-\gamma_{-}}(\bar{x})\right| \leq c_{1} d(\bar{x}, \Gamma)
\end{array}
$$

due to the same kind of relation as (26), but written for $-\gamma_{-}$instead of $\gamma_{-}$. Returning back to (31), we see that

$$
\frac{1}{c_{1}} d(\bar{x}, \Gamma) \leq\left|F^{\gamma_{+}}(\hat{x})\right| \times\left|\gamma_{-}\left(\pi_{\Gamma}(\hat{x})\right)\right|=\left|\tilde{F}^{\gamma_{+}}(\hat{x})\right| \frac{\left|\gamma_{-}\left(\pi_{\Gamma}(\hat{x})\right)\right|}{\left|\gamma_{+}\left(\pi_{\Gamma}(\hat{x})\right)\right|} \leq c_{1} d(\bar{x}, \Gamma) .
$$

So that in view of (26) written for $\hat{x}$ and $\gamma_{+}$,

$$
\frac{1}{c_{1}^{2}} \frac{\left|\gamma_{+}\left(\pi_{\Gamma}(\hat{x})\right)\right|}{\left|\gamma_{-}\left(\pi_{\Gamma}(\hat{x})\right)\right|} d(\bar{x}, \Gamma) \leq d(\hat{x}, \Gamma) \leq c_{1}^{2} \frac{\left|\gamma_{+}\left(\pi_{\Gamma}(\hat{x})\right)\right|}{\left|\gamma_{-}\left(\pi_{\Gamma}(\hat{x})\right)\right|} d(\bar{x}, \Gamma) .
$$

But using (6) and (7), it easy to see that for any $z \in \Gamma$,

$$
\frac{\lambda^{2}}{\Lambda^{2} d^{2}} \leq \frac{\left|\gamma_{+}(z)\right|^{2}}{\left|\gamma_{-}(z)\right|^{2}} \leq \frac{\Lambda^{2} d^{2}}{\lambda^{2}}
$$

from which we deduce the result of the proposition.

\subsection{Our transformed Euler scheme}

We are now in position to introduce our transformed Euler scheme.

Let us denote from now on $\triangle t=h_{n}=\frac{T}{n}$ the time step (where $n \in \mathbb{N}^{*}$ ) and fix a starting point $x_{0} \in \mathbb{R}^{d}$.

The time grid is given by $\left(t_{k}^{n}\right)_{k=0}^{n}$ with $t_{k}^{n}=\frac{T k}{n}$ for $0 \leq k \leq n$.

We denote by $\left(\Delta W_{k+1}\right)_{k=0}^{n}$ the i.i.d. sequence of Brownian increments constructed on $\left(\Omega, \mathcal{F}, \mathbb{P}^{x_{0}}\right)$ and defined by

$$
\Delta W_{k+1}=W_{t_{k+1}}-W_{t_{k}}, \quad \forall 0 \leq k \leq n .
$$




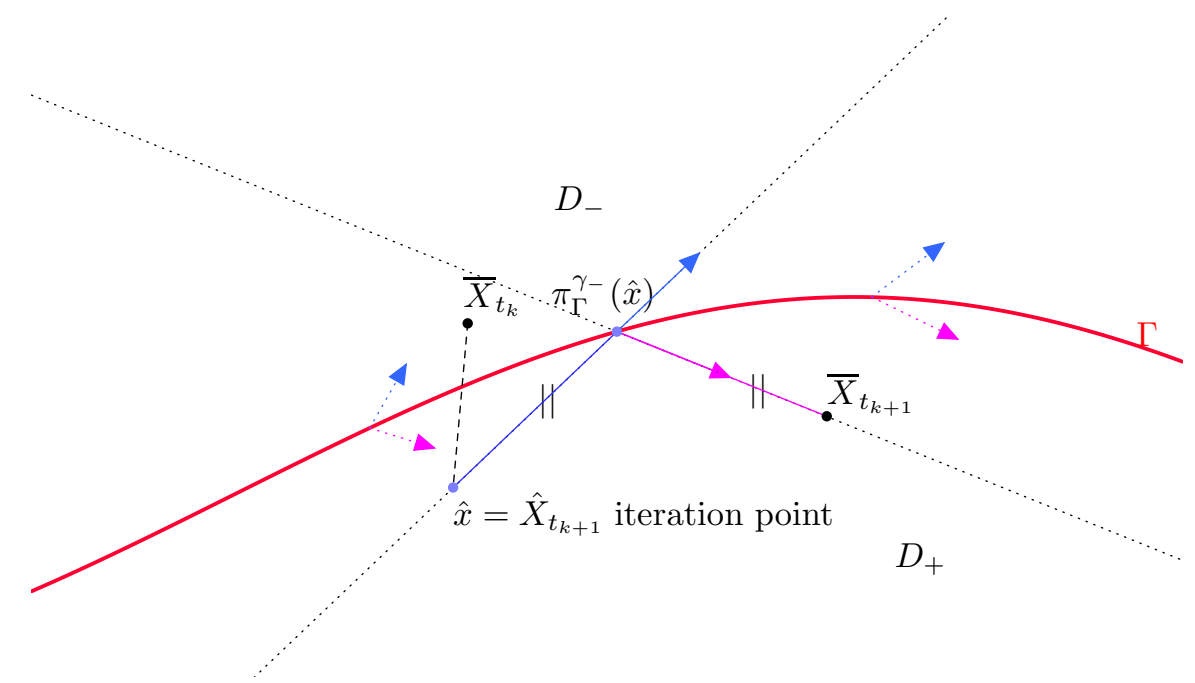

Figure 1: Correction of our scheme when the path crosses the boundary $\Gamma$.

Recall that $\sigma: \mathbb{R}^{d} \rightarrow \mathbb{R}^{d \times d}$ stands for a matrix valued coefficient satisfying

$$
\sigma \sigma^{*}(x)=2 a(x), \quad \forall x \in D .
$$

Set $(\partial a(x))_{j}=\operatorname{div}\left(x \mapsto\left(a_{1 j}(x), \ldots, a_{n j}(x)\right)\right)$.

Our stochastic numerical scheme $\left(\bar{X}_{t_{k}}^{n}\right)_{k=0}^{n}$ is defined as follows (we omit the superscript $n$ )

$$
\bar{X}_{0}=x_{0}
$$

and for $t \in\left(t_{k}, t_{k+1}\right]$, we set

$$
\left\{\begin{array}{rr}
\hat{X}_{t}=\bar{X}_{t_{k}}+\sigma\left(\bar{X}_{t_{k}}\right)\left(W_{t}-W_{t_{k}}\right)+\partial a\left(\bar{X}_{t_{k}}\right)\left(t-t_{k}\right) & \text { (standard Euler incrementation) } \\
\bar{X}_{t_{k+1}}=\hat{X}_{t_{k+1}} & \text { if }\left(\bar{X}_{t_{k}} \in \bar{D}_{+} \text {and } \hat{X}_{t_{k+1}} \in D_{+}\right) \\
& \text {or }\left(\bar{X}_{t_{k}} \in \bar{D}_{-} \text {and } \hat{X}_{t_{k+1}} \in D_{-}\right) ; \\
\bar{X}_{t_{k+1}}=\pi_{\Gamma}^{\gamma_{+}}\left(\hat{X}_{t_{k+1}}\right)-F^{\gamma_{+}}\left(\hat{X}_{t_{k+1}}\right) \gamma_{-}\left(\pi_{\Gamma}^{\gamma_{+}}\left(\hat{X}_{t_{k+1}}\right)\right) & \text { if } \bar{X}_{t_{k}} \in \bar{D}_{+} \text {and } \hat{X}_{t_{k+1}} \in D_{-} ; \\
\bar{X}_{t_{k+1}}=\pi_{\Gamma}^{\gamma-}\left(\hat{X}_{t_{k+1}}\right)-F^{\gamma_{-}}\left(\hat{X}_{t_{k+1}}\right) \gamma_{+}\left(\pi_{\Gamma}^{\gamma-}\left(\hat{X}_{t_{k+1}}\right)\right) & \text { if } \bar{X}_{t_{k}} \in \bar{D}_{-} \text {and } \hat{X}_{t_{k+1}} \in D_{+} .
\end{array}\right.
$$

Remark 4.2. Note that by its construction the presented Euler scheme enjoys uniqueness in law. 
Remark 4.3. (Comparison with known results in the classical smooth case)

- Note that whenever a is smooth, the co-normal vector fields $\gamma^{+}$and $\gamma^{-}$ are in exactly opposite directions and our problem becomes artificial.

Combining (32) with (24) and (25) shows that the construction of our scheme ensures that $\hat{X}_{t_{k}}=\bar{X}_{t_{k}}$ for $k=0, \ldots, n$ in this particular and artificial case (see also Figure 1 and observe that the isosceles triangle becomes flat in this case). Thus, there is no correction when crossing the boundary and - whenever a is smooth - our scheme reduces the classical Euler scheme.

- Note also that at the crossing of the boundary our construction resembles the so-called symmetrized Euler scheme constructed for the specific case of reflected diffusions (see [5]). However in [5] (see also [19]) the purpose of the construction is to forbid the numerical scheme from crossing the reflecting boundary (without undermining the convergence), contrary to our framework where we let the scheme cross freely the transmission boundary (the correction point remains in the same side as its corresponding iteration point). Up to our current knowledge both problems - reflected boundary problem and transmission boundary problem - cannot be treated in a common framework. Still, we feel that both problems share the same flavor and the reader might want to compare the picture of Figure 1 with Figure 1 p. 881 in [5].

Remark 4.4. (Comparison with the results of [37] in the one dimensional case)

When the dimension $d$ is reduced to 1 (one dimensional problem), the discontinuity surface reduces to a single point (say 0). In this case and when the coefficient $a=a_{+} \mathbb{I}_{y>0}+a_{-} \mathbb{I}_{y<0}$ is constant on both sides of the discontinuity, it is remarkable that our Euler Scheme is exactly the same as the one described in [37].

Indeed, in this one-dimensional context, let $\varphi(y)=\left(a_{-} \mathbb{I}_{y>0}+a_{+} \mathbb{I}_{y<0}\right) y$. Note that $\varphi$ is a bijective map from $\mathbb{R}$ to $\mathbb{R}$. The Euler Scheme constructed in [37] is then defined by $\bar{X}_{0}=x_{0}$ and for all $k \in\{0, \ldots, n\}$,

$$
\bar{X}_{t_{k}}=\varphi^{-1}\left(\bar{Y}_{t_{k}}\right)
$$

where $\bar{Y}_{0}=\varphi\left(x_{0}\right)$ and for all $k \in\{0, \ldots, n-1\}$

$$
\bar{Y}_{t_{k+1}}=\bar{Y}_{t_{k}}+\left(a_{-} \sigma_{+} \mathbb{I}_{\bar{Y}_{t_{k}}>\mathrm{o}}+a_{+} \sigma_{-} \mathbb{I}_{\bar{Y}_{t_{k}}<\mathrm{o}}\right)\left(W_{t_{k+1}}-W_{t_{k}}\right) ;
$$

(see [37] for details - please take care that [37] is written for the right-hand sided local time; the above computation is valid for the symmetric local time). For example if $\varphi\left(\bar{X}_{t_{k}}\right)<0$ and $\bar{Y}_{t_{k+1}} \geq 0$, we get (because $\varphi^{-1}(0)=0$ and $\varphi^{-1}$ 
is continuous at 0 and also because $\bar{X}$ and $\bar{Y}$ share the same sign),

$$
\begin{aligned}
\bar{X}_{t_{k+1}}= & \varphi^{-1}\left(\varphi\left(\bar{X}_{t_{k}}\right)+\left(a_{-} \sigma_{+} \mathbb{I}_{\bar{Y}_{t_{k}}>\mathrm{o}}+a_{+} \sigma_{-} \mathbb{I}_{\bar{Y}_{t_{k}}<\mathrm{o}}\right) \Delta W_{k}^{k+1}\right) \\
= & \bar{X}_{t_{k}}+\int_{\varphi\left(\bar{X}_{t_{k}}\right)}^{0}\left(\varphi^{-1}\right)^{\prime}(z) d z \\
& \quad+\int_{0}^{\varphi\left(\bar{X}_{t_{k}}\right)+(\underbrace{a_{-} \sigma_{+} \mathbb{I}_{\bar{Y}_{t_{k}}>0}}_{=0}+a_{+} \sigma_{-} \mathbb{I}_{\bar{Y}_{t_{k}}<0}) \Delta W_{k}^{k+1}}\left(\varphi^{-1}\right)^{\prime}(z) d z \\
= & \bar{X}_{t_{k}}-\varphi\left(\bar{X}_{t_{k}}\right) \frac{1}{a_{+}}+\left(\varphi\left(\bar{X}_{t_{k}}\right)+a_{+} \sigma_{-} \Delta W_{k}^{k+1}\right) \frac{1}{a_{-}} \\
= & \frac{a_{+}}{a_{-}} \bar{X}_{t_{k}}+\frac{a_{+}}{a_{-}} \sigma_{-} \Delta W_{k}^{k+1},
\end{aligned}
$$

which turns out to be the corresponding case stated in (32) in this one-dimensional context. This correspondence is valid in all the other cases.

Hence, our transformed Euler Scheme may be viewed as some kind of multidimensional generalization of the Euler Scheme presented in [37] (up to second order terms if the coefficient a of the operator is not piece-wise constant).

\section{Convergence rate of our Euler scheme}

The purpose of this section is to prove the following result.

Theorem 5.1. Let $0<T<\infty$. Assume (E). Let $m^{\prime}=\left\lceil 2+\frac{d}{4}\right\rceil+1$ and $k=m^{\prime}+2$. Assume that the coefficients $a_{i j}$ satisfy $\left(a_{ \pm}\right)_{i j} \in C_{b}^{2 m^{\prime}-1}\left(\bar{D}_{ \pm}\right)$and that $\Gamma$ is of class $C^{2 m^{\prime}}$. Let $u_{0}: \mathbb{R}^{d} \rightarrow \mathbb{R}$ be in the space $\mathcal{D}\left(A^{k}\right)$. Let $u$ be a classical solution of $\left(\mathcal{P}_{\mathrm{T}}\right)$.

We have that for all $n$ large enough,

$$
\sup _{x_{0} \in \mathbb{R}^{d}}\left|u\left(T, x_{0}\right)-\mathbb{E}^{x_{0}} u_{0}\left(\bar{X}_{T}^{n}\right)\right| \leq K \sqrt{h_{n}},
$$

where the constant $K$ depends on $d, \lambda, \Lambda, u_{0}$ and $T$.

As a consequence of the above and under these assumptions, there is uniqueness of the classical solution $u$ of $\left(\mathcal{P}_{\mathrm{T}}\right)$.

Remark 5.1. Note that the uniqueness of $u$ appears as a consequence of the uniqueness in law of our numerical scheme (Remark 4.2) and of the convergence (33). This is in the spirit of the last statement of Theorem 5.7.6 in [21] where the uniqueness of the solution of parabolic PDEs is proved by the mean of a Feynman-Kac representation formula involving a diffusion $X$ which enjoys uniqueness in law.

Remark 5.2. In Theorem 5.1 the assumptions on $a(x)$ and $\Gamma$ involving the integers $m^{\prime}$ and $k$ are here in order to use Corollary 3.2, which ensures that we will have $\sup _{t \in[0, T], x \in \bar{D}_{ \pm}}\left|\partial_{t}^{j} \partial^{\alpha} u_{ \pm}(t, x)\right|<\infty$ for any $j \leq 2$ and any $|\alpha| \leq$ 
4. This control on the derivatives on $u$ is what we need in order to lead our convergence proof. In fact if there is a way to get this control under weaker assumptions on $a(x)$ and $\Gamma$ our methodology of proof would lead to a convergence theorem stated under these weaker assumptions.

The rate of convergence in Theorem 5.1 is of order $\sqrt{h_{n}}$. This order is much slower than the order $h_{n}$ achieved for the standard Euler scheme applied to SDEs with smooth coefficients (see [47] and [2]). However this rate $\sqrt{h_{n}}$ improves very slightly the results obtained in [36]. This is due to the methodology of proof where we manage to use smooth discounted occupation times for the scheme around the interface instead of discretized occupation times involving harsh indicator functions.

Unfortunately we have not been able to prove a second order transmission boundary condition as the one derived in Lemma 3 p. 883 in [5] for the case of the symmetrized Euler scheme for reflected diffusions. This explains the reason why we do not achieve the rate $h_{n}$ as in [5]. Note that the rate $\sqrt{h_{n}}$ seems confirmed by numerical 1D tests performed in [35] and is classical for transmission problems.

Note that similarly to the results obtained for the symmetrized Euler scheme for reflected diffusions in [5], our convergence result holds uniformly with respect to the starting point $x_{0}$.

In the sequel of this section we focus on proving (33).

\subsection{Preliminary results}

Lemma 5.1. (see [5] Lemma 1 p. 883)

Consider an Itô process with uniformly bounded coefficients $d U_{t}=b_{t} d t+$ $\sigma_{t} d W_{t}$ on $(\Omega, \mathcal{F}, \mathbb{P})$. There exist some constants $c>0$ and $K$ (depending on $p \geq 1, T$ and the bounds on $\sigma, b)$ such that, for any stopping times $S$ and $S^{\prime}$ (with $0 \leq S \leq S^{\prime} \leq \delta \leq T$ ) and any $\eta \geq 0$,

$$
\begin{aligned}
& \mathbb{P}\left[\sup _{t \in\left[S, S^{\prime}\right]}\left|U_{t}-U_{s}\right| \geq \eta\right] \leq K \exp \left(-c \frac{\eta^{2}}{\delta}\right) ; \\
& \mathbb{E}\left[\sup _{t \in\left[S, S^{\prime}\right]}\left|U_{t}-U_{s}\right|^{p}\right] \leq K \delta^{p / 2} .
\end{aligned}
$$

We have when $\bar{X}_{t_{k}} \in D_{+}$

$$
\bar{X}_{t_{k+1}}=\hat{X}_{t_{k+1}}+\left[F^{\gamma_{+}}\left(\hat{X}_{t_{k+1}}\right)\right]^{-}\left(\gamma_{+}\left(\pi_{\Gamma}^{\gamma_{+}}\left(\hat{X}_{t_{k+1}}\right)\right)+\gamma_{-}\left(\pi_{\Gamma}^{\gamma_{+}}\left(\hat{X}_{t_{k+1}}\right)\right)\right)
$$

and when $X_{t_{k}} \in D_{-}$

$$
\bar{X}_{t_{k+1}}=\hat{X}_{t_{k+1}}+\left[F^{\gamma_{-}}\left(\hat{X}_{t_{k+1}}\right)\right]^{-}\left(\gamma_{+}\left(\pi_{\Gamma}^{\gamma-}\left(\hat{X}_{t_{k+1}}\right)\right)+\gamma_{-}\left(\pi_{\Gamma}^{\gamma_{-}}\left(\hat{X}_{t_{k+1}}\right)\right)\right)
$$


This shows that $\left(\bar{X}_{t}\right)_{0 \leq t \leq T}$ behaves like a continuous semimartingale on each of the intervals $\left[t_{k}, t_{k+1}\right)$.

We now aim at finding the differential form for $\left(\bar{X}_{t}\right)_{0 \leq t \leq T}$.

Using Tanaka's formula (remember that we are using the symmetric local time), we have

$$
\begin{aligned}
d\left[F^{\gamma_{+}}\left(\hat{X}_{t}\right)\right]^{-}= & -\int_{0}^{t} \mathbb{I}_{F^{\gamma_{+}}\left(\hat{X}_{t}\right)<\mathrm{o}} d\left(F^{\gamma_{+}}\left(\hat{X}_{t}\right)\right)+\frac{1}{2} d L_{t}^{0}\left(F^{\gamma_{+}}(\hat{X})\right) \\
= & -\int_{0}^{t} \mathbb{I}_{F^{\gamma_{+}}\left(\hat{X}_{t}\right)<\mathrm{o}} \nabla F^{\gamma_{+}}\left(\hat{X}_{t}\right) d \hat{X}_{t}-\int_{0}^{t} \mathbb{I}_{F^{\gamma_{+}}\left(\hat{X}_{t}\right)<\mathrm{o}} \frac{1}{2} \operatorname{Tr}\left[\mathbf{H}\left[F^{\gamma_{+}}\right]\left(\hat{X}_{t}\right) a\left(\bar{X}_{t_{k}}\right)\right] d t \\
& +\frac{1}{2} d L_{t}^{0}\left(F^{\gamma_{+}}(\hat{X})\right) .
\end{aligned}
$$

Moreover, using Remark 4.1 we may and will write that

$$
\left(\gamma_{+}\left(\pi_{\Gamma}^{\gamma_{+}}\left(\hat{X}_{t_{k+1}}\right)\right)+\gamma_{-}\left(\pi_{\Gamma}^{\gamma_{+}}\left(\hat{X}_{t_{k+1}}\right)\right)\right)=\left(\gamma_{+}\left(\hat{X}_{t_{k+1}}\right)+\gamma_{-}\left(\hat{X}_{t_{k+1}}\right)\right)
$$

which allows to apply Itô's formula to $\left(\hat{X}_{t}\right)_{t_{k}<t \leq t_{k+1}}$ along the vector fields $\gamma_{+}$ and $\gamma_{-}$without having to differentiate the co-normal projections.

Hence, applying these differentiations when $\bar{X}_{t_{k}} \in D_{+}$we find that for any $t \in\left[t_{k}, t_{k+1}\right)$,

$$
\begin{aligned}
& d \bar{X}_{t}=d \hat{X}_{t}+\frac{\left(\gamma_{+}+\gamma_{-}\right)}{2}\left(\hat{X}_{t}\right) d L_{t}^{0}\left(F^{\gamma_{+}}(\hat{X})\right)+\left[F^{\gamma_{+}}\left(\hat{X}_{t}\right)\right]^{-}\left(\nabla\left(\gamma_{+}+\gamma_{-}\right)\left(\hat{X}_{t}\right) d \hat{X}_{t}\right. \\
& \left.+\frac{1}{2} \operatorname{Tr}\left[\mathbf{H}\left[\gamma_{+}+\gamma_{-}\right]\left(\hat{X}_{t}\right) a\left(\bar{X}_{t_{k}}\right)\right] d t\right) \\
& -\mathbb{I}_{F^{\gamma_{+}\left(\hat{X}_{t}\right)<\mathrm{o}}}\left[\nabla\left(\gamma_{+}+\gamma_{-}\right)\left(\hat{X}_{t}\right) a\left(\bar{X}_{t_{k}}\right)\left(\nabla F^{\gamma_{+}}\left(\hat{X}_{t}\right)\right)^{*} d t\right. \\
& \left.+\left(\gamma_{+}+\gamma_{-}\right)\left(\hat{X}_{t}\right) \nabla F^{\gamma_{+}}\left(\hat{X}_{t}\right) d \hat{X}_{t}\right] \\
& -\mathbb{I}_{F^{\gamma_{+}}\left(\hat{X}_{t}\right)<\mathrm{o}}\left(\gamma_{+}+\gamma_{-}\right)\left(\hat{X}_{t}\right) \frac{1}{2} \operatorname{Tr}\left[\mathbf{H}\left[F^{\gamma_{+}}\right]\left(\hat{X}_{t}\right) a\left(\bar{X}_{t_{k}}\right)\right] d t
\end{aligned}
$$

and a corresponding equality holds also true for $\bar{X}_{t_{k}} \in D_{-}$.

Lemma 5.2. Under the assumptions of Theorem 5.1, for all $c>0$, there exists a constant $K(T)$ such that

$$
h_{n} \mathbb{E}^{x_{0}} \sum_{i=0}^{n-1}\left[\exp \left(-c \frac{d^{2}\left(\bar{X}_{t_{i}}^{n}, \Gamma\right)}{h_{n}}\right)\right] \leq K(T) \sqrt{h_{n}}
$$

uniformly over $x_{0} \in \mathbb{R}^{d}$.

Proof. The idea is to use the occupation times formula. Using successively (26) and the inequality (30) of Proposition 4.2, we have $d(\bar{x}, \Gamma) \geq \frac{1}{c_{2}} d(\hat{x}, \Gamma) \geq$ 


$$
\begin{aligned}
& \frac{1}{c_{1} c_{2}}\left|\tilde{F}^{\gamma_{ \pm}}(\hat{x})\right| \text { so that } \\
& \mathcal{A}_{i+1}:=\mathbb{E}^{x_{0}}\left[\exp \left(-c \frac{d^{2}\left(\bar{X}_{t_{i+1}}^{n}, \Gamma\right)}{h_{n}}\right)\right] \\
& \leq \mathbb{E}^{x_{0}}\left[\exp \left(-c \frac{\left|\tilde{F}^{\gamma_{+}}\left(\hat{X}_{t_{i+1}}^{n}\right)\right|^{2}}{\left|c_{1} c_{2}\right|^{2} h_{n}}\right) \mathbb{I}_{\bar{X}_{t_{i+1}}^{n} \in D_{-}}\right] \\
& +\mathbb{E}^{x_{0}}\left[\exp \left(-c \frac{\left|\tilde{F}^{\gamma-}\left(\hat{X}_{t_{i+1}}^{n}\right)\right|^{2}}{\left|c_{1} c_{2}\right|^{2} h_{n}}\right) \mathbb{I}_{\bar{X}_{t_{i+1}}^{n} \in D_{+}}\right] \\
& :=\mathcal{A}_{i+1}^{+}+\mathcal{A}_{i+1}^{-} \text {. }
\end{aligned}
$$

We concentrate on term $\mathcal{A}_{i+1}^{+}$as both terms are treated in a similar manner.

Set $c^{\prime}=c / 2 c_{1}^{2} c_{2}^{2}>0$ and $g(x)=\exp \left(-2 c^{\prime} x^{2} / h\right)$; it is easy to check that $|g(x)|+\sqrt{h}\left|g^{\prime}(x)\right|+h\left|g^{\prime \prime}(x)\right| \leq K(T) \exp \left(-c^{\prime} x^{2} / h\right)$. Hence, for $t \in\left[t_{i}, t_{i+1}\right]$, Itô's formula yields that

$$
\begin{aligned}
& \mathbb{E}^{x_{0}} \exp \left(-2 c^{\prime} \frac{\left|\tilde{F}^{\gamma_{+}}\left(\hat{X}_{t_{i+1}}^{n}\right)\right|^{2}}{h_{n}}\right) \\
& \leq K(T)\left[\mathbb{E}^{x_{0}} \exp \left(-c^{\prime} \frac{\left|\tilde{F}^{\gamma_{+}}\left(\hat{X}_{t}^{n}\right)\right|^{2}}{h_{n}}\right)\right. \\
& \left.\quad+\frac{1}{h_{n}} \int_{t}^{t_{i+1}} d s \mathbb{E}^{x_{0}} \exp \left(-c^{\prime} \frac{\left|\tilde{F}^{\gamma_{+}}\left(\hat{X}_{s}^{n}\right)\right|^{2}}{h_{n}}\right)\right] .
\end{aligned}
$$

We integrate this inequality with respect to $t$ over $\left[t_{i}, t_{i+1}\right]$ to get

$$
h_{n} \mathcal{A}_{i+1}^{+} \leq K(T) \int_{t_{i}}^{t_{i+1}} d s \mathbb{E}^{x_{0}} \exp \left(-c^{\prime} \frac{\left|\tilde{F}^{\gamma_{+}}\left(\hat{X}_{s}^{n}\right)\right|^{2}}{h_{n}}\right) .
$$

(for possibly some new constant $K(T)$ ).

Observe that from (27),

$$
d\left\langle\tilde{F}^{\gamma_{+}}\left(\hat{X}^{n}\right), \tilde{F}^{\gamma_{+}}\left(\hat{X}^{n}\right)\right\rangle_{s}=\nabla \tilde{F}^{\gamma_{+}}\left(\hat{X}_{s}^{n}\right) a\left(\bar{X}_{t_{i}}^{n}\right)\left[\nabla \tilde{F}^{\gamma_{+}}\left(\hat{X}_{s}^{n}\right)\right]^{*} d s \geq \lambda d s .
$$

Indeed, using the Cauchy-Schwarz inequality and $|\nu(\hat{x})|=1$, we have that

$$
\begin{aligned}
\nabla \tilde{F}^{\gamma_{+}}(\hat{x}) a(\bar{x})\left[\nabla \tilde{F}^{\gamma_{+}}(\hat{x})\right]^{*} & =\frac{\nu^{*}(\hat{x}) a(\bar{x}) \nu(\hat{x})}{\left\langle\nu(\hat{x}), \tilde{\gamma}_{+}(\hat{x})\right\rangle^{2}}=\frac{\langle\nu(\hat{x}), a(\bar{x}) \nu(\hat{x})\rangle}{\left\langle\nu(\hat{x}), \frac{a(\hat{x}) \nu(\hat{x})}{|a(\hat{x}) \nu(\hat{x})|}\right\rangle^{2}} \\
& \geq \frac{\langle\nu(\hat{x}), a(\bar{x}) \nu(\hat{x})\rangle}{|\nu(\hat{x})|^{2}|a(\hat{x}) \nu(\hat{x})|^{2}}|a(\hat{x}) \nu(\hat{x})|^{2}=\langle\nu(\hat{x}), a(\bar{x}) \nu(\hat{x})\rangle \\
& \geq \lambda
\end{aligned}
$$


which justifies (40).

It readily follows from the occupation times formula that

$$
h_{n} \mathcal{A}_{i+1}^{+} \leq K(T) \int_{-R}^{R} d y \exp \left(-c^{\prime} \frac{y^{2}}{h_{n}}\right) \mathbb{E}^{x_{0}}\left[\triangle_{i}^{i+1} L^{y}\left(\tilde{F}^{\gamma_{+}}\left(\hat{X}_{.}^{n}\right)\right)\right] .
$$

Now,

$$
\begin{aligned}
& \mathbb{E}^{x_{0}}\left[L_{t_{i+1}}^{y}\left(\tilde{F}^{\gamma_{+}}\left(\hat{X}_{.}^{n}\right)\right)-L_{t_{i}}^{y}\left(\tilde{F}^{\gamma_{+}}\left(\hat{X}_{.}^{n}\right)\right)\right] \\
& =2 \mathbb{E}^{x_{0}}\left[\left(\tilde{F}^{\gamma_{+}}\left(\hat{X}_{t_{i+1}}^{n}\right)-y\right)^{+}-\left(\tilde{F}^{\gamma_{+}}\left(\hat{X}_{t_{i}}^{n}\right)-y\right)^{+}\right. \\
& \left.\quad-\int_{t_{i}}^{t_{i+1}} \mathbb{I}_{\tilde{F}^{\gamma_{+}}\left(\hat{X}_{s}^{n}\right) \geq y} d\left(\tilde{F}^{\gamma_{+}}\left(\hat{X}_{s}^{n}\right)\right)\right] \\
& \leq 2 \mathbb{E}^{x_{0}}\left[\left(\tilde{F}^{\gamma_{+}}\left(\hat{X}_{t_{i+1}}^{n}\right)-y\right)^{+}-\left(\tilde{F}^{\gamma_{+}}\left(\hat{X}_{t_{i}}^{n}\right)-y\right)^{+}\right]+K(T) h_{n} .
\end{aligned}
$$

Therefore, $\sum_{i=0}^{n-1} \mathbb{E}^{x_{0}}\left[L_{t_{i+1}}^{y}\left(\tilde{F}^{\gamma_{+}}\left(\hat{X}_{.}^{n}\right)\right)-L_{t_{i}}^{y}\left(\tilde{F}^{\gamma_{+}}\left(\hat{X}_{.}^{n}\right)\right)\right] \leq K(T)$ uniformly in $|y| \leq R$ since the sum is telescoping. Moreover, the result of Lemma 5.1 ensures that this inequality is uniform w.r.t $x_{0}$. We can thus conclude that $h_{n} \sum_{i=0}^{n-1} \mathcal{A}_{i+1}^{+} \leq K(T) \sqrt{h_{n}}$ uniformly w.r.t $x_{0}$.

The sum $h_{n} \sum_{i=0}^{n-1} \mathcal{A}_{i+1}^{-}$is treated similarly. The proof of the Lemma is complete.

\subsection{Error decomposition}

In all the sequel $x_{0}$ is arbitrarily fixed.

For all $0 \leq k \leq n$ set

$$
\theta_{k}^{n}:=T-t_{k}^{n}
$$

The proof of Theorem 5.1 proceeds as follows (we omit the superscript $n$ ). Since $u(0, x)=u_{0}(x)$ for all $x \in \mathbb{R}^{d}$ and $u\left(T, x_{0}\right)=\mathbb{E}^{x_{0}} u\left(T, \bar{X}_{0}\right)$, the discretization error at time $T$ can be decomposed as follows:

$$
\begin{aligned}
\epsilon_{T}^{x_{0}} & =\left|u\left(T, x_{0}\right)-\mathbb{E}^{x_{0}} u_{0}\left(\bar{X}_{T}\right)\right| \\
& =\left|\sum_{k=0}^{n-1} \mathbb{E}^{x_{0}} u\left(T-t_{k}, \bar{X}_{t_{k}}\right)-\mathbb{E}^{x_{0}} u\left(T-t_{k+1}, \bar{X}_{t_{k+1}}\right)\right|,
\end{aligned}
$$

and thus

$$
\begin{aligned}
\epsilon_{T}^{x_{0}} \leq \mid \sum_{k=0}^{n-1} \mathbb{E}^{x_{0}}\left\{u\left(\theta_{k}, \bar{X}_{t_{k}}\right)-u\left(\theta_{k+1}, \bar{X}_{t_{k}}\right)\right. \\
\left.+u\left(\theta_{k+1}, \bar{X}_{t_{k}}\right)-u\left(\theta_{k+1}, \bar{X}_{t_{k+1}}\right)\right\} \mid .
\end{aligned}
$$

The rest of this section is devoted to the analysis of

$$
\left|\sum_{k=0}^{n-1} \mathbb{E}^{x_{0}}\left(T_{k}-S_{k}\right)\right|,
$$


where the time increment $T_{k}$ is defined as

$$
T_{k}:=u\left(\theta_{k}, \bar{X}_{t_{k}}\right)-u\left(\theta_{k+1}, \bar{X}_{t_{k}}\right)
$$

and the space increment is defined as

$$
S_{k}:=u\left(\theta_{k+1}, \bar{X}_{t_{k+1}}\right)-u\left(\theta_{k+1}, \bar{X}_{t_{k}}\right) .
$$

\subsection{Estimate for the time increment $T_{k}$}

Remember the definition (44) of $T_{k}$ and that $\theta_{k}=T-t_{k}$. We have

$$
\begin{aligned}
& \left\{u\left(\theta_{k}, \bar{X}_{t_{k}}\right)-u\left(\theta_{k+1}, \bar{X}_{t_{k}}\right)\right\} \mathbb{I}_{\bar{X}_{t_{k}} \in D_{+}} \\
& =h_{n} \partial_{t} u\left(\theta_{k+1}, \bar{Y}_{t_{k}}\right) \mathbb{I}_{\bar{X}_{t_{k}} \in D_{+}} \\
& +h_{n}^{2} \int_{[0,1]^{2}} \partial_{t t}^{2} u\left(\theta_{k+1}+\alpha_{1} \alpha_{2} h_{n}, \bar{X}_{t_{k}}\right) \alpha_{1} d \alpha_{1} d \alpha_{2} \mathbb{I}_{\bar{X}_{t_{k}} \in D_{+}} \\
& =: T_{k}^{+}+R_{k}^{+} \text {. }
\end{aligned}
$$

Similarly,

$$
\begin{aligned}
& \left\{u\left(\theta_{k}, \bar{X}_{t_{k}}\right)-u\left(\theta_{k+1}, \bar{X}_{t_{k}}\right)\right\} \mathbb{I}_{\bar{X}_{t_{k}} \in D_{-}} \\
& =h_{n} \partial_{t} u\left(\theta_{k+1}, \bar{X}_{t_{k}}\right) \mathbb{I}_{\bar{X}_{t_{k}} \in D_{-}} \\
& +h_{n}^{2} \int_{[0,1]^{2}} \partial_{t t}^{2} u\left(\theta_{k+1}+\alpha_{1} \alpha_{2} h_{n}, \bar{X}_{t_{k}}\right) \alpha_{1} d \alpha_{1} d \alpha_{2} \mathbb{I}_{\bar{X}_{t_{k}} \in D_{-}} \\
& =: T_{k}^{-}+R_{k}^{-} \text {. }
\end{aligned}
$$

In view of Corollary 3.2 and Remark 5.2 we have

$$
\mathbb{E}^{x_{0}}\left|R_{k}^{+}+R_{k}^{-}\right| \leq C h_{n}^{2} .
$$

From the preceding we deduce

$$
\mathbb{E}^{x_{0}} T_{k}=\mathbb{E}^{x_{0}} \partial_{t} u\left(\theta_{k+1}, \bar{X}_{t_{k}}\right) h_{n}+O\left(h_{n}^{2}\right) .
$$

\subsection{Expansion of the space increment $S_{k}$}

Let $S_{k}$ be defined as in (45). Set

$$
\begin{aligned}
\triangle_{k+1} \bar{X} & :=\bar{X}_{t_{k+1}}-\bar{X}_{t_{k}} \\
\triangle_{k+1}^{\sharp} \bar{X} & :=\hat{X}_{t_{k+1}}-\bar{X}_{t_{k}} .
\end{aligned}
$$

and recall that $\triangle_{k+1} W=W_{t_{k+1}}-W_{t_{k}}$.

Proposition 5.1.

$$
\mathbb{E}^{x_{0}}\left|\left(\triangle_{k+1}^{\sharp} \bar{X}\right)^{\alpha}\right| \leq C(\alpha) h_{n}^{|\alpha| / 2} .
$$

Proof. This is a consequence of the result of Lemma 5.1 combined with the fact that $\left|(x)^{\alpha}\right| \leq|x|^{|\alpha|}$ for any $x \in \mathbb{R}^{d}$. 
We emphasize that, due to the definition of our stochastic scheme, $\triangle_{k+1}^{\sharp} \bar{X}$ does not coincide with $\bar{X}_{t_{k+1}}-\bar{X}_{t_{k}}$ when $\bar{X}_{t_{k+1}}$ and $\bar{X}_{t_{k}}$ do not belong to the same region, which explains the two notations $\triangle$ and $\triangle^{\sharp}$.

We need to introduce the four following events:

$$
\begin{cases}\Omega_{k}^{++} & :=\left[\bar{X}_{t_{k}} \in \bar{D}_{+} \text {and } \hat{X}_{t_{k+1}} \in D_{+}\right], \\ \Omega_{k}^{--} & :=\left[\bar{X}_{t_{k}} \in D_{-} \text {and } \hat{X}_{t_{k+1}} \in \bar{D}_{-}\right], \\ \Omega_{k}^{+-} & :=\left[\bar{X}_{t_{k}} \in D_{+} \text {and } \hat{X}_{t_{k+1}} \in \bar{D}_{-}\right] \\ \Omega_{k}^{-+} & :=\left[\bar{X}_{t_{k}} \in \bar{D}_{-} \text {and } \hat{X}_{t_{k+1}} \in D_{+}\right]\end{cases}
$$

In view of the definition of our stochastic numerical scheme we have

$$
\text { On } \Omega_{k}^{++}, \triangle_{k+1} \bar{X}=\triangle_{k+1}^{\sharp} \bar{X} \text {. }
$$

Therefore

$$
\begin{aligned}
S_{k} \mathbb{I}_{\Omega_{k}^{++}}= & \left\langle\triangle_{k+1} \bar{X}, \nabla_{x} u\left(\theta_{k+1}, \bar{X}_{t_{k}}\right)\right\rangle \mathbb{I}_{\Omega_{k}^{++}} \\
+ & \frac{1}{2}\left(\triangle_{k+1} \bar{X}\right)^{*} \mathbf{H}[u]\left(\theta_{k+1}, \bar{X}_{t_{k}}\right) \triangle_{k+1} \bar{X} \mathbb{I}_{\Omega_{k}^{++}} \\
& +\sum_{|\alpha|=3} \frac{1}{\alpha !}\left(\triangle_{k+1} \bar{X}\right)^{\alpha} \frac{\partial^{3} u}{\partial x^{\alpha}}\left(\theta_{k+1}, \bar{X}_{t_{k}}\right) \mathbb{I}_{\Omega_{k}^{++}} \\
& +\int_{0}^{1} d \xi \sum_{|\alpha|=4} \frac{(1-\xi)^{4}}{\alpha !}\left(\triangle_{k+1} \bar{X}\right)^{\alpha} \frac{\partial^{4} u}{\partial x^{\alpha}}\left(\theta_{k+1}, \bar{X}_{t_{k}}+\xi \triangle_{k+1} \bar{X}\right) \mathbb{I}_{\Omega_{k}^{++}} \\
= & : S_{k}^{++1}+S_{k}^{++2}+S_{k}^{++3}+S_{k}^{++4} .
\end{aligned}
$$

Similarly,

$$
\begin{aligned}
S_{k} \mathbb{I}_{\Omega_{k}^{--}}= & \left\langle\triangle_{k+1} \bar{X}, \nabla_{x} u\left(\theta_{k+1}, \bar{X}_{t_{k}}\right)\right\rangle \mathbb{I}_{\Omega_{k}^{--}} \\
+ & \frac{1}{2}\left(\triangle_{k+1} \bar{X}\right)^{*} \mathbf{H}[u]\left(\theta_{k+1}, \bar{X}_{t_{k}}\right) \triangle_{k+1} \bar{X} \mathbb{I}_{\Omega_{k}^{--}} \\
& +\sum_{|\alpha|=3} \frac{1}{\alpha !}\left(\triangle_{k+1} \bar{X}\right)^{\alpha} \frac{\partial^{3} u}{\partial x^{\alpha}}\left(\theta_{k+1}, \bar{X}_{t_{k}}\right) \mathbb{I}_{\Omega_{k}^{--}} \\
& +\int_{0}^{1} d \xi \sum_{|\alpha|=4} \frac{(1-\xi)^{4}}{\alpha !}\left(\triangle_{k+1} \bar{X}\right)^{\alpha} \frac{\partial^{4} u}{\partial x^{\alpha}}\left(\theta_{k+1}, \bar{X}_{t_{k}}+\xi \triangle_{k+1} \bar{X}\right) \mathbb{I}_{\Omega_{k}^{--}} \\
= & : S_{k}^{--1}+S_{k}^{--2}+S_{k}^{--3}+S_{k}^{--4} .
\end{aligned}
$$

We now use that $\Omega_{k}^{++} \cup \Omega_{k}^{--}=\Omega-\left(\Omega_{k}^{+-} \cup \Omega_{k}^{-+}\right)$. Notice that $\Omega_{k}^{+-} \cup \Omega_{k}^{-+}$ belongs to the $\sigma$-field generated by $\left(W_{t}\right)$ up to time $t_{k+1}$. In view of the first line of (32) and the fact that $\mathbb{E}^{\mathcal{F}_{t_{k}}} \Delta W_{k+1}=0$, we get

$$
\begin{aligned}
\mathbb{E}^{x_{0}}\left(S_{k}^{++1}+S_{k}^{--1}\right)= & \frac{h_{n}}{2} \mathbb{E}^{x_{0}}\left[\left\langle\partial a\left(\bar{X}_{t_{k}}\right), \nabla_{x} u\left(\theta_{k+1}, \bar{X}_{t_{k}}\right)\right\rangle\right] \\
& -\mathbb{E}^{x_{0}}\left[\left\langle\triangle_{k+1}^{\sharp} \bar{X}, \nabla_{x} u\left(\theta_{k+1}, \bar{X}_{t_{k}}\right)\right\rangle \mathbb{I}_{\Omega_{k}^{+-} \cup \Omega_{k}^{-+}}\right] .
\end{aligned}
$$


Proceeding similarly and conditioning $\left(\triangle_{k+1}^{\sharp} \bar{X}\right)^{2}$ w.r.t. the past of $\left(W_{t}\right)$ up to time $t_{k}$, we obtain

$$
\begin{aligned}
\mathbb{E}^{x_{0}}\left(S_{k}^{++2}+S_{k}^{--2}\right)= & \frac{1}{2} \mathbb{E}^{x_{0}}\left[\operatorname{Tr}\left[\sigma \mathbf{H}[u] \sigma^{*}\right]\left(\theta_{k+1}, \bar{X}_{t_{k}}\right)\right] h_{n} \\
& -\frac{1}{2} \mathbb{E}^{x_{0}}\left[\left(\triangle_{k+1}^{\sharp} \bar{X}\right)^{*} \mathbf{H}[u]\left(\theta_{k+1}, \bar{X}_{t_{k}}\right) \triangle_{k+1}^{\sharp} \bar{X} \mathbb{I}_{\Omega_{k}^{+-} \cup \Omega_{k}^{-+}}\right]
\end{aligned}
$$

and since $\mathbb{E}^{x_{0}}\left(\triangle_{k+1} W\right)^{\alpha}=0$ whenever $|\alpha|=3$,

$$
\begin{aligned}
\mathbb{E}^{x_{0}}\left(S_{k}^{++3}+S_{k}^{--3}\right)= & \sum_{|\alpha|=3} \frac{1}{\alpha !} \mathbb{E}^{x_{0}}\left[\left(\triangle_{k+1}^{\sharp} \bar{X}\right)^{\alpha} \frac{\partial^{3} u}{\partial x^{\alpha}}\left(\theta_{k+1}, \bar{X}_{t_{k}}\right)\right] \\
& -\sum_{|\alpha|=3} \frac{1}{\alpha !} \mathbb{E}^{x_{0}}\left[\left(\triangle_{k+1}^{\sharp} \bar{X}\right)^{\alpha} \frac{\partial^{3} u}{\partial x^{\alpha}}\left(\theta_{k+1}, \bar{X}_{t_{k}}\right) \mathbb{I}_{\Omega_{k}^{+-} \cup \Omega_{k}^{-+}}\right] .
\end{aligned}
$$

We have, combining the results of Corollary 3.2 and Proposition 5.1,

$$
\left|\sum_{|\alpha|=3} \frac{1}{\alpha !} \mathbb{E}^{x_{0}}\left[\left(\triangle_{k+1}^{\sharp} \bar{X}\right)^{\alpha} \frac{\partial^{3} u}{\partial x^{\alpha}}\left(\theta_{k+1}, \bar{X}_{t_{k}}\right)\right]\right| \leq C h_{n}^{3 / 2} .
$$

In addition, and for the same reasons, we have

$$
\mathbb{E}^{x_{0}}\left|S_{k}^{++4}+S_{k}^{--4}\right| \leq C h_{n}^{2} .
$$

To summarize the calculations of this subsection, we have obtained

$$
\begin{aligned}
& \mathbb{E}^{x_{0}} S_{k}= \\
& \mathbb{E}^{x_{0}} \mathcal{L} u\left(\theta_{k+1}, \bar{X}_{t_{k}}\right) h_{n}+\mathbb{E}^{x_{0}}\left[\left(S_{k}-\left\langle\triangle_{k+1}^{\sharp} \bar{X}, \nabla_{x} u\left(\theta_{k+1}, \bar{X}_{t_{k}}\right)\right\rangle\right) \mathbb{I}_{\Omega_{k}^{+-} \cup \Omega_{k}^{-+}}\right] \\
& -\mathbb{E}^{x_{0}}\left[\left(\frac{1}{2}\left(\triangle_{k+1}^{\sharp} \bar{X}\right)^{*} \mathbf{H}[u]\left(\theta_{k+1}, \bar{X}_{t_{k}}\right) \triangle_{k+1}^{\sharp} \bar{X}\right.\right. \\
& \left.\left.+\sum_{|\alpha|=3} \frac{1}{\alpha !}\left(\triangle_{k+1}^{\sharp} \bar{X}\right)^{\alpha} \frac{\partial^{3} u}{\partial x^{\alpha}}\left(\theta_{k+1}, \bar{X}_{t_{k}}\right)\right) \mathbb{I}_{\Omega_{k}^{+-} \cup \Omega_{k}^{-+}}\right]+O\left(h_{n}^{3 / 2}\right) \\
& =: \mathbb{E}^{x_{0}} \mathcal{L} u\left(\theta_{k+1}, \bar{X}_{t_{k}}\right) h_{n}+\mathbb{E}^{x_{0}} \mathcal{R}_{k}^{(1)}-\mathbb{E}^{x_{0}} \mathcal{R}_{k}^{(2)}+O\left(h_{n}^{3 / 2}\right) .
\end{aligned}
$$

We now estimate the remaining terms $\mathbb{E}^{x_{0}} \mathcal{R}_{k}^{(1)}$ and $\mathbb{E}^{x_{0}} \mathcal{R}_{k}^{(2)}$.

5.5. Control of the term $\mathbb{E}^{x_{0}} \mathcal{R}_{k}^{(1)}$. Expansion around a well chosen point in $\Gamma$

On the event $\Omega_{k}^{+-}$we have that $\bar{X}_{t_{k+1}}$ and $\bar{X}_{t_{k}}$ are close to $\Gamma$. On this event, we also have that $\hat{X}_{t_{k+1}} \in D_{-}$and $\bar{X}_{t_{k}} \in D_{+}$. Remember our definition of $\left(F^{\gamma_{+}}(x), \pi_{\Gamma}^{\gamma_{+}}(x)\right)$ for $x \in D_{-}$. 


\subsubsection{Decomposition of $\mathbb{E}^{x_{0}} \mathcal{R}_{k}^{(1)}$}

As the function $u$ is continuous across the surface $\Gamma$ at point $\pi_{\Gamma}^{\gamma_{+}}(x)$, we get

$$
\begin{aligned}
& \mathbb{E}^{x_{0}}\left(\left(S_{k}-\left\langle\triangle_{k+1}^{\sharp} \bar{X}, \nabla_{x} u\left(\theta_{k+1}, \bar{X}_{t_{k}}\right)\right\rangle\right) \mathbb{I}_{\Omega_{k}^{+-}}\right] \\
& =\mathbb{E}^{x_{0}}\left[\left(\left(u\left(\theta_{k+1}, \bar{X}_{t_{k+1}}\right)-u\left(\theta_{k+1}, \pi_{\Gamma}^{\gamma^{+}}\left(\hat{X}_{t_{k+1}}\right)\right)\right)\right.\right. \\
& \left.\left.+\left(u\left(\theta_{k+1}, \pi_{\Gamma}^{\gamma_{+}}\left(\hat{X}_{t_{k+1}}\right)\right)-u\left(\theta_{k+1}, \bar{X}_{t_{k}}\right)\right)\right) \mathbb{I}_{\Omega_{k}^{+-}}\right] \\
& \quad-\mathbb{E}^{x_{0}}\left[\left\langle\triangle_{k+1}^{\sharp} \bar{X}, \nabla_{x} u_{+}\left(\theta_{k+1}, \pi_{\Gamma}^{\gamma_{+}}\left(\hat{X}_{t_{k+1}}\right)\right)\right\rangle \mathbb{I}_{\Omega_{k}^{+-}}\right] \\
& \quad-\mathbb{E}^{x_{0}}\left[\left\langle\triangle_{k+1}^{\sharp} \bar{X}, \nabla_{x} u\left(\theta_{k+1}, \bar{X}_{t_{k}}\right)-\nabla_{x} u_{+}\left(\theta_{k+1}, \pi_{\Gamma}^{\gamma_{+}}\left(\hat{X}_{t_{k+1}}\right)\right)\right\rangle \mathbb{I}_{\Omega_{k}^{+-}}\right]
\end{aligned}
$$

so that

$$
\begin{aligned}
& \mathbb{E}^{x_{0}}\left(\left(S_{k}-\left\langle\triangle_{k+1}^{\sharp} \bar{X}, \nabla_{x} u\left(\theta_{k+1}, \bar{X}_{t_{k}}\right)\right\rangle\right) \mathbb{I}_{\Omega_{k}^{+-}}\right] \\
& =L_{k}^{+-1}+L_{k}^{+-2}+L_{k}^{+-3},
\end{aligned}
$$

where

$$
\begin{aligned}
& L_{k}^{+-1}:=\left\{\mathbb{E}^{x_{0}}\left[\left\langle\bar{X}_{t_{k+1}}-\pi_{\Gamma}^{\gamma+}\left(\hat{X}_{t_{k+1}}\right), \nabla_{x} u_{-}\left(\theta_{k+1}, \pi_{\Gamma}^{\gamma_{+}}\left(\hat{X}_{t_{k+1}}\right)\right)\right\rangle \mathbb{I}_{\Omega_{k}^{+-}}\right]\right. \\
&- \mathbb{E}^{x_{0}}\left[\left\langle\bar{X}_{t_{k}}-\pi_{\Gamma}^{\gamma+}\left(\hat{X}_{t_{k+1}}\right), \nabla_{x} u_{+}\left(\theta_{k+1}, \pi_{\Gamma}^{\gamma_{+}}\left(\hat{X}_{t_{k+1}}\right)\right)\right\rangle \mathbb{I}_{\Omega_{k}^{+-}}\right] \\
&-\left.\mathbb{E}^{x_{0}}\left[\left\langle\triangle_{k+1}^{\sharp} \bar{X}, \nabla_{x} u_{+}\left(\theta_{k+1}, \pi_{\Gamma}^{\gamma+}\left(\hat{X}_{t_{k+1}}\right)\right)\right\rangle \mathbb{I}_{\Omega_{k}^{+-}}\right]\right\} \\
& L_{k}^{+-2}:=\left\{\int _ { 0 } ^ { 1 } d \xi \sum _ { | \alpha | = 2 } \frac { ( 1 - \xi ) ^ { 2 } } { \alpha ! } \mathbb { E } ^ { x _ { 0 } } \left[\left(\hat{X}_{t_{k+1}}-\pi_{\Gamma}^{\gamma+}\left(\hat{X}_{t_{k+1}}\right)\right)^{\alpha}\right.\right. \\
&\left.\quad \times \frac{\partial^{\alpha} u}{\partial x^{\alpha}}\left(\theta_{k+1}, \pi_{\Gamma}^{\gamma_{+}}\left(\hat{X}_{t_{k+1}}\right)+\xi\left(\hat{X}_{t_{k+1}}-\pi_{\Gamma}^{\gamma_{+}}\left(\hat{X}_{t_{k+1}}\right)\right)\right) \mathbb{I}_{\Omega_{k}^{+-}}\right] \\
&-\int_{0}^{1} d \xi \sum_{|\alpha|=2} \frac{(1-\xi)^{2}}{\alpha !} \mathbb{E}^{x_{0}}\left[\left(\bar{X}_{t_{k}}-\pi_{\Gamma}^{\gamma_{+}}\left(\hat{X}_{t_{k+1}}\right)\right)^{\alpha}\right. \\
&\left.\left.\times \frac{\partial^{\alpha} u}{\partial x^{\alpha}}\left(\theta_{k+1}, \pi_{\Gamma}^{\gamma_{+}}\left(\hat{X}_{t_{k+1}}\right)+\xi\left(\bar{X}_{t_{k}}-\pi_{\Gamma}^{\gamma_{+}}\left(\hat{X}_{t_{k+1}}\right)\right)\right) \mathbb{I}_{\Omega_{k}^{+-}}\right]\right\}
\end{aligned}
$$

and

$$
L_{k}^{+-3}:=-\left\{\mathbb{E}^{x_{0}}\left[\left\langle\triangle_{k+1}^{\sharp} \bar{X}, \nabla_{x} u_{+}\left(\theta_{k+1}, \bar{X}_{t_{k}}\right)-\nabla_{x} u_{+}\left(\theta_{k+1}, \pi_{\Gamma}^{\gamma_{+}}\left(\hat{X}_{t_{k+1}}\right)\right)\right\rangle \mathbb{I}_{\Omega_{k}^{+-}}\right]\right\} .
$$

5.5.2. Canceling the term $L_{k}^{+-1}$ using the transmission condition

Observe that due to the fact that

$$
\left(\hat{X}_{t_{k}}-\pi_{\Gamma}^{\gamma_{+}}\left(\hat{X}_{t_{k+1}}\right)\right)+\left(\hat{X}_{t_{k+1}}-\hat{X}_{t_{k}}\right)=\hat{X}_{t_{k+1}}-\pi_{\Gamma}^{\gamma_{+}}\left(\hat{X}_{t_{k+1}}\right) .
$$


we have that

$$
\begin{gathered}
L_{k}^{+-1}=\mathbb{E}^{x_{0}}\left[\left(\left\langle\bar{X}_{t_{k+1}}-\pi_{\Gamma}^{\gamma+}\left(\hat{X}_{t_{k+1}}\right), \nabla_{x} u_{-}\left(\theta_{k+1}, \pi_{\Gamma}^{\gamma_{+}}\left(\hat{X}_{t_{k+1}}\right)\right)\right\rangle\right.\right. \\
\left.\left.\quad-\left\langle\hat{X}_{t_{k+1}}-\pi_{\Gamma}^{\gamma+}\left(\hat{X}_{t_{k+1}}\right), \nabla_{x} u_{+}\left(\theta_{k+1}, \pi_{\Gamma}^{\gamma+}\left(\hat{X}_{t_{k+1}}\right)\right)\right\rangle\right) \mathbb{I}_{\Omega_{k}^{+-}}\right] \\
=\mathbb{E}^{x_{0}}\left[F ^ { \gamma + } ( \hat { X } _ { t _ { k + 1 } } ) \left(\left\langle-\gamma_{-}\left(\pi_{\Gamma}^{\gamma+}\left(\hat{X}_{t_{k+1}}\right)\right), \nabla_{x} u_{-}\left(\theta_{k+1}, \pi_{\Gamma}^{\gamma+}\left(\hat{X}_{t_{k+1}}\right)\right)\right\rangle\right.\right. \\
\left.\left.\quad-\left\langle\gamma_{+}\left(\pi_{\Gamma}^{\gamma+}\left(\hat{X}_{t_{k+1}}\right)\right), \nabla_{x} u_{+}\left(\theta_{k+1}, \pi_{\Gamma}^{\gamma+}\left(\hat{X}_{t_{k+1}}\right)\right)\right\rangle\right) \mathbb{I}_{\Omega_{k}^{+-}}\right] \\
=0,
\end{gathered}
$$

where we have used the vector problem solved by $\left(F^{\gamma_{+}}, \pi_{\Gamma}^{\gamma_{+}}\right)$and Equation (28) (i.e. the transmission condition $(\star)$ and the definition of $\gamma_{ \pm}(x)$ ).

\subsubsection{The term $L_{k}^{+-2}$}

We now turn to the term $L_{k}^{+-2}$.

The term $L_{k}^{+-2}$ is the sum of two terms. These two terms are treated similarly, so we concentrate only on the first. Let $\alpha$ such that $|\alpha|=2$. We have that

$$
\begin{aligned}
\mathbb{E}^{x_{0}}\left[\left|\left(\bar{X}_{t_{k}}-\pi_{\Gamma}^{\gamma_{+}}\left(\hat{X}_{t_{k+1}}\right)\right)^{\alpha}\right| \mathbb{I}_{\Omega_{k}^{+-}}\right] & \leq c_{1} \mathbb{E}^{x_{0}}\left[\left|\bar{X}_{t_{k}}-\pi_{\Gamma}^{\gamma_{+}}\left(\hat{X}_{t_{k+1}}\right)\right|^{2} \mathbb{I}_{\Omega_{k}^{+-}}\right] \\
& \leq c_{2} \mathbb{E}^{x_{0}}\left[\left|\triangle_{k+1}^{\sharp} \bar{X}\right|^{2} \mathbb{I}_{\Omega_{k}^{+-}}\right] .
\end{aligned}
$$

The same kind of treatment can be performed for the second term of $L_{k}^{+-2}$. Conditionning w.r.t $\mathcal{F}_{t_{k}}$ and applying the Cauchy-Schwarz inequality in the conditionnal expectation, we find using the result of Lemma 5.1,

$$
\begin{aligned}
\left|L_{k}^{+-2}\right| & \leq C \mathbb{E}^{x_{0}}\left[\mathbb{E}^{\mathcal{F}_{t_{k}}}\left[\left|\triangle_{k+1}^{\sharp} \bar{X}\right|^{4}\right]^{1 / 2} \mathbb{P}^{\mathcal{F}_{t_{k}}}\left(\Omega_{k}^{+-}\right)^{1 / 2}\right] \\
& \leq C h_{n} \mathbb{E}^{x_{0}} \mathbb{P}^{\mathcal{F}_{t_{k}}}\left(\Omega_{k}^{+-}\right)^{1 / 2} .
\end{aligned}
$$

\subsubsection{The term $L_{k}^{+-3}$}

For the term $L_{k}^{+-3}$, we may perform a Taylor's expansion to the term

$$
\nabla_{x} u_{+}\left(\theta_{k+1}, \bar{X}_{t_{k}}\right)-\nabla_{x} u_{+}\left(\theta_{k+1}, \pi_{\Gamma}^{\gamma_{+}}\left(\hat{X}_{t_{k+1}}\right)\right) .
$$

Using Corollary 3.2 and the Cauchy-Schwarz inequality, we find that

$$
\begin{aligned}
\left|L_{k}^{+-3}\right| & \leq C \mathbb{E}^{x_{0}}\left[\left|\triangle_{k+1}^{\sharp} \bar{X}\right|\left|\bar{X}_{t_{k}}-\pi_{\Gamma}^{\gamma+}\left(\hat{X}_{t_{k+1}}\right)\right\rangle \mid \mathbb{I}_{\Omega_{k}^{+-}}\right] \\
& \leq C \mathbb{E}^{x_{0}}\left[\left|\triangle_{k+1}^{\sharp} \bar{X}\right|^{2} \mathbb{I}_{\Omega_{k}^{+-}}\right] .
\end{aligned}
$$

Finally, as for the term $L_{k}^{+-2}$, we find that

$$
\left|L_{k}^{+-3}\right| \leq C h_{n} \mathbb{E}^{x_{0}} \mathbb{P}^{\mathcal{F}_{t_{k}}}\left(\Omega_{k}^{+-}\right)^{1 / 2} .
$$

Using the same method for the other side $\Omega_{k}^{-+}$, we find that

$$
\mathbb{E}^{x_{0}} \mathcal{R}_{k}^{(1)} \leq C h_{n} \mathbb{E}^{x_{0}}\left(\mathbb{P}^{\mathcal{F}_{t_{k}}}\left(\Omega_{k}^{+-}\right)^{1 / 2}+\mathbb{P}^{\mathcal{F}_{t_{k}}}\left(\Omega_{k}^{-+}\right)^{1 / 2}\right) .
$$




\subsection{Summing up}

The term $\mathbb{E}^{x_{0}} \mathcal{R}_{k}^{(2)}$ can be estimated using the same techniques used in the previous section and we omit the details.

Using now the fact that $\partial_{t} u-\mathcal{L} u=0$, we finally find that

$$
\epsilon_{T}^{x_{0}} \leq C h_{n} \mathbb{E}^{x_{0}} \sum_{k=0}^{n-1}\left(\mathbb{P}^{\mathcal{F}_{t_{k}}}\left(\Omega_{k}^{+-}\right)^{1 / 2}+\mathbb{P}^{\mathcal{F}_{t_{k}}}\left(\Omega_{k}^{-+}\right)^{1 / 2}\right)+C \sqrt{h_{n}} .
$$

Observe - using the result of Lemma 5.1 - that

$$
\begin{aligned}
\mathbb{P}^{\mathcal{F}_{t_{k}}}\left(\Omega_{k}^{+-}\right)^{1 / 2} & =\mathbb{P}^{\mathcal{F}_{t_{k}}}\left(\bar{X}_{t_{k}} \in D_{+}, \hat{X}_{t_{k+1}} \in D_{-}\right)^{1 / 2} \\
& \leq \mathbb{P}^{\mathcal{F}_{t_{k}}}\left(\left\|\hat{X}_{t_{k+1}}-\bar{X}_{t_{k}}\right\| \geq d\left(\bar{X}_{t_{k}}, \Gamma\right)\right)^{1 / 2} \\
& \leq K(T) \exp \left(-\frac{1}{2} \frac{d^{2}\left(\bar{X}_{t_{k}}, \Gamma\right)}{h_{n}}\right)
\end{aligned}
$$

and the same kind of inequality holds true for $\mathbb{P}^{\mathcal{F}_{t_{k}}}\left(\Omega_{k}^{-+}\right)^{1 / 2}$.

Finally,

$$
\epsilon_{T}^{x_{0}} \leq K(T) h_{n} \mathbb{E}^{x_{0}} \sum_{k=0}^{n-1} \exp \left(-\frac{1}{2} \frac{d^{2}\left(\bar{X}_{t_{k}}, \Gamma\right)}{h_{n}}\right)+C \sqrt{h_{n}},
$$

and we conclude the proof of Theorem 5.1 using the result of Lemma 5.2 (note that if we sum up all the dependancies of our constants, we indeed have that $K$ in (33) depends on $d, \lambda, \Lambda, u_{0}$ and $T$ ).

\section{Numerical experiments}

In the forthcoming Examples 1 and 2 we have $d=2$ and consider PDEs in a bounded spatial domain $D_{0}=D_{+} \cup D_{-} \cup \Gamma$ to be given by the open unit disc, i.e.,

$$
D_{0}=\left\{\left(x_{1}, x_{2}\right) \in \mathbb{R}: x_{1}^{2}+x_{2}^{2}<1\right\} .
$$

The boundary of $D_{0}$ is thus the unit circle $\partial D_{0}=\left\{\left(x_{1}, x_{2}\right) \in \mathbb{R}: x_{1}^{2}+x_{2}^{2}=1\right\}$.

The subdomains $D_{+}$and $D_{-}$are defined by

$$
D_{+}=\left\{\left(x_{1}, x_{2}\right) \in D \text { with } x_{2}>0\right\} \quad \text { and } \quad D_{-}=\left\{\left(x_{1}, x_{2}\right) \in D \text { with } x_{2}<0\right\} \text {, }
$$

so that the interface is $\Gamma=\left\{\left(x_{1}, 0\right) \in \mathbb{R}^{2}:-1 \leq x_{1} \leq 1\right\}$. We recall the notation $D=D_{+} \cup D_{-}$.

Note that the forthcoming parabolic problem $\left(\mathcal{P}_{\mathrm{T}, \text { bounded }} D_{0}\right)$ of Example 2 is then posed in a bounded domain, unlike in our theoretical study. But we have found that convenient for numerical purposes.

The diffusion matrix is defined by

$$
a(x)=a_{+}(x) \mathbb{I}_{x \in D_{+}}+a_{-}(x) \mathbb{I}_{x \in \bar{D}_{-}},
$$


with

$$
a_{ \pm}(x)=P_{ \pm}^{*} E_{ \pm}(x) P_{ \pm}
$$

where $P_{ \pm}$are rotation (therefore orthogonal) matrices

$$
P_{ \pm}=\left(\begin{array}{cc}
\cos \left(\theta_{ \pm}\right) & -\sin \left(\theta_{ \pm}\right) \\
\sin \left(\theta_{ \pm}\right) & \cos \left(\theta_{ \pm}\right)
\end{array}\right)
$$

(for $\theta_{ \pm} \in[0,2 \pi)$ ), and $E_{ \pm}(x)$ are diagonal matrix-valued functions

$$
E_{ \pm}(x)=\left(\begin{array}{cc}
\lambda_{ \pm}^{1}+\epsilon_{ \pm} x_{2} & 0 \\
0 & \lambda_{ \pm}^{2}+\epsilon_{ \pm} x_{2}
\end{array}\right)
$$

where $\lambda_{ \pm}^{1}, \lambda_{ \pm}^{2}>0$ and $\epsilon_{ \pm}<\lambda_{ \pm}^{i}$ for $i=1,2$. Note that this ensures that $a(x)$ satisfies the uniform ellipticity assumption $(\mathbf{E})$.

We take $\theta_{+}=\frac{\pi}{4}, \theta_{-}=\frac{\pi}{3}, \lambda_{+}^{1}=1, \lambda_{+}^{2}=9, \lambda_{-}^{1}=2, \lambda_{-}^{2}=3, \epsilon_{+}=0.5$ and $\epsilon_{-}=1.9$. This gives

$$
a_{+}(x)=\frac{1}{2}\left(\begin{array}{cc}
5+0.5 x_{2} & 4 \\
4 & 5+0.5 x_{2}
\end{array}\right), a_{-}(x)=\frac{1}{2}\left(\begin{array}{cc}
\frac{11}{4}+1.9 x_{2} & \frac{\sqrt{3}}{4} \\
\frac{\sqrt{3}}{4} & \frac{9}{4}+1.9 x_{2}
\end{array}\right) .
$$

\section{Performing our Transformed Euler Scheme.}

We have the Cholesky decompositions $2 a_{ \pm}(x)=\sigma_{ \pm} \sigma_{ \pm}^{*}(x)$, with

$$
\sigma_{+}(x)=\left(\begin{array}{cc}
\sqrt{5+0.5 x_{2}} & 0 \\
4 / \sqrt{5+0.5 x_{2}} & \sqrt{5+0.5 x_{2}-16 /\left(5+0.5 x_{2}\right)}
\end{array}\right)
$$

and

$$
\sigma_{-}(x)=\left(\begin{array}{cc}
\sqrt{\frac{11}{4}+1.9 x_{2}} & 0 \\
\frac{\sqrt{3}}{4} / \sqrt{\frac{11}{4}+1.9 x_{2}} & \sqrt{\frac{9}{4}+1.9 x_{2}-3 /\left(44+30.4 x_{2}\right)}
\end{array}\right),
$$

so that $2 a(x)=\sigma \sigma^{*}(x)$ with $\sigma(x)=\sigma_{+}(x) \mathbb{I}_{x \in D_{+}}+\sigma_{-}(x) \mathbb{I}_{x \in \bar{D}_{-}}$. Besides we have

$$
\partial a(x)=\left(\begin{array}{c}
0 \\
0.25
\end{array}\right) \mathbb{I}_{x \in D_{+}}+\left(\begin{array}{c}
0 \\
0.95
\end{array}\right) \mathbb{I}_{x \in \bar{D}_{-}} .
$$

Note that when the scheme crosses the interface $\Gamma$, we compute the quantities $\pi_{\Gamma}^{\gamma_{ \pm}}\left(\hat{X}_{t_{k+1}}\right)$ and $F^{\gamma_{ \pm}}\left(\hat{X}_{t_{k+1}}\right)$ in the following way (we will detail the procedure for $\pi_{\Gamma}^{\gamma_{+}}\left(\hat{X}_{t_{k+1}}\right)$ and $\left.F^{\gamma_{+}}\left(\hat{X}_{t_{k+1}}\right)\right)$. Recall that we have

$$
\hat{X}_{t_{k+1}}-\pi_{\Gamma}^{\gamma_{+}}\left(\hat{X}_{t_{k+1}}\right)=F^{\gamma_{+}}\left(\hat{X}_{t_{k+1}}\right) \gamma_{+}\left(\pi_{\Gamma}^{\gamma_{+}}\left(\hat{X}_{t_{k+1}}\right)\right) .
$$

But here $\nu=(0,1)^{*}$ so that for any $x \in \Gamma$

$$
\gamma_{+}(x)=\frac{1}{2}\left(\begin{array}{c}
4 \\
5+0.5 x_{2}
\end{array}\right)
$$


and $\left(\pi_{\Gamma}^{\gamma+}\left(\hat{X}_{t_{k+1}}\right)\right)_{2}=0$ so that $\left(\hat{X}_{t_{k+1}}-\pi_{\Gamma}^{\gamma+}\left(\hat{X}_{t_{k+1}}\right)\right)_{2}=\left(\hat{X}_{t_{k+1}}\right)_{2}$. This yields

$$
F^{\gamma_{+}}\left(\hat{X}_{t_{k+1}}\right)=\frac{\left(\hat{X}_{t_{k+1}}\right)_{2}}{2.5}
$$

and then

$$
\pi_{\Gamma}^{\gamma+}\left(\hat{X}_{t_{k+1}}\right)=\left(\begin{array}{c}
\left(\hat{X}_{t_{k+1}}\right)_{1}-F^{\gamma_{+}}\left(\hat{X}_{t_{k+1}}\right) \times 2 \\
0
\end{array}\right) .
$$

Then we have everything in hand to perform our Tranformed Euler Scheme $\bar{X}$.

Comparing with an Euler scheme applied on regularized coefficients. A natural method with which to compare our tranformed scheme is to regularize first the coefficients and then to perform a standard (i.e. not transformed) Euler scheme. More precisely consider the operator

$$
C^{2}\left(\mathbb{R}^{d} ; \mathbb{R}\right) \ni f \mapsto \mathcal{L}^{\varepsilon} f=\nabla \cdot\left(a^{\varepsilon} \nabla_{x} f\right)=\operatorname{Tr}\left[\mathbf{H}[f] a^{\varepsilon}\right]+\left(\partial a^{\varepsilon}\right)^{*} \nabla_{x} f
$$

where $a^{\varepsilon}$ is some smoothed version of $a$ ( $\varepsilon$ is the regularization step, see the following discussion about its choice). Then $\mathcal{L}^{\varepsilon}$ is the generator of the solution of the SDE

$$
d X_{t}^{\varepsilon}=\sigma^{\varepsilon}\left(X_{t}^{\varepsilon}\right) d W_{t}+\left[\partial a^{\varepsilon}\right]\left(X_{t}^{\varepsilon}\right) d t,
$$

where $\sigma^{\varepsilon}\left(\sigma^{\varepsilon}\right)^{*}=2 a^{\varepsilon}$. The process $X^{\varepsilon}$ may be approached by a standard (i.e. not transformed) Euler scheme $\bar{X}^{\varepsilon}$, with time step $h_{n}$.

Let $h_{n}$ be fixed. In fact $\varepsilon$ will be chosen in function of $h_{n}$. We are first inspired by the random walk approach proposed in [46]. In this later paper Equation (3.11) indicated that $\varepsilon$ has to be proportional to the square root of the space discretisation step. Then, using a scaling argument we choose $\varepsilon=h_{n}^{1 / 4}$.

Then we set

$$
a^{\varepsilon}(x)=a(x) \mathbf{1}_{\left|x_{2}\right|>\varepsilon}+A^{\varepsilon}(x) \mathbf{1}_{\left|x_{2}\right| \leq \varepsilon}
$$

where

$$
A^{\varepsilon}(x)=\frac{1}{2}\left(\begin{array}{cc}
\frac{31}{8}-0.7 \varepsilon+x_{2}\left(\frac{9}{8 \varepsilon}+1.2\right) & \frac{\sqrt{3}}{8}+2+x_{2}\left(\frac{2}{\varepsilon}-\frac{\sqrt{3}}{8 \varepsilon}\right) \\
\frac{\sqrt{3}}{8}+2+x_{2}\left(\frac{2}{\varepsilon}-\frac{\sqrt{3}}{8 \varepsilon}\right) & \frac{29}{8}-0.7 \varepsilon+x_{2}\left(\frac{11}{8 \varepsilon}+1.2\right)
\end{array}\right) .
$$

Note that the thus defined coefficient $a^{\varepsilon}$ is continuous and piecewise differentiable. Then we have $\partial a^{\varepsilon}=\partial a(x) \mathbf{1}_{\left|x_{2}\right|>\varepsilon}+\partial A^{\varepsilon}(x) \mathbf{1}_{\left|x_{2}\right| \leq \varepsilon}$ where

$$
\partial A^{\varepsilon}(x)=\left(\begin{array}{c}
\frac{1}{\varepsilon}-\frac{\sqrt{3}}{16 \varepsilon} \\
\frac{11}{16 \varepsilon}+0.6
\end{array}\right),
$$

and $2 a^{\varepsilon}(x)=\sigma^{\varepsilon}\left[\sigma^{\varepsilon}\right]^{*}(x)$ with $\sigma^{\varepsilon}(x)=\sigma(x) \mathbf{1}_{\left|x_{2}\right|>\varepsilon}+\Sigma^{\varepsilon}(x) \mathbf{1}_{\left|x_{2}\right| \leq \varepsilon}$ and $\Sigma^{\varepsilon}(x)$ being equal to

$$
\left(\begin{array}{cc}
\sqrt{\frac{31}{8}-0.7 \varepsilon+x_{2}\left(\frac{9}{8 \varepsilon}+1.2\right)} & 0 \\
\frac{\frac{\sqrt{3}}{8}+2+x_{2}\left(\frac{2}{\varepsilon}-\frac{\sqrt{3}}{8 \varepsilon}\right)}{\sqrt{\frac{31}{8}-0.7 \varepsilon+x_{2}\left(\frac{9}{8 \varepsilon}+1.2\right)}} & \sqrt{\frac{29}{8}-0.7 \varepsilon+x_{2}\left(\frac{11}{8 \varepsilon}+1.2\right)-\frac{\left(\frac{\sqrt{3}}{8}+2+x_{2}\left(\frac{2}{\varepsilon}-\frac{\sqrt{3}}{8 \varepsilon}\right)\right)^{2}}{\frac{31}{8}-0.7 \varepsilon+x_{2}\left(\frac{9}{8 \varepsilon}+1.2\right)}}
\end{array}\right)
$$


With these coefficients it is easy to perform a standard Euler Scheme on the SDE (53).

We will compare both methods on the two following examples. Benchmarks will be provided by a deterministic approximation of the solutions of the PDE of interest.

Example 1. We wish here to treat the elliptic transmission problem

$$
\left(\mathcal{E}_{\mathrm{T}, \text { bounded } D_{0}}^{0}\right)\left\{\begin{array}{rlrl}
\mathcal{L} v(x) & =0 & & \forall x \in D \\
\left\langle a_{+} \nabla_{x} v_{+}(y)-a_{-} \nabla_{x} v_{-}(y), \nu(y)\right\rangle & =0 & \forall y \in \Gamma \\
v(y+) & =v(y-) & \forall y \in \Gamma \\
v(x) & =f(x) & \forall x \in \partial D_{0} .
\end{array}\right.
$$

We take the function $f$ to be

$$
f(x)=\sin \left(3 x_{1}\right)+\cos \left(4 x_{2}\right) .
$$

Consider then on one side our study of the convergence in the parabolic case, and on the other side the Feynman-Kac representation for elliptic PDEs available in the smooth case (see for instance Theorem 5.7.2 in [21]). One can hope that

$$
\mathbb{E}^{x}\left[f\left(\bar{X}_{\bar{\tau}}\right)\right] \underset{h_{n} \rightarrow 0}{\longrightarrow} v(x),
$$

where $\bar{X}$ denotes our scheme and $\bar{\tau}=\inf \left\{t \geq 0: \bar{X}_{t} \notin D_{0}\right\}$.

We thus compute a Monte Carlo approximation of $\mathbb{E}^{x}\left[f\left(\bar{X}_{\bar{\tau}}\right)\right]$ on one side (with $N=10^{6}$ paths). Note that in this Monte Carlo procedure we have used a boundary shifting method, on order to reduce the bias introduced by the approximation of the exit time $\tau=\inf \left\{t \geq 0: X_{t} \notin D_{0}\right\}$ by $\bar{\tau}$ (see [20] Subsection 5.4.3, and the references therein).

On the other side $\mathbb{E}^{x}\left[f\left(\bar{X}_{\bar{\tau}^{\varepsilon}}^{\varepsilon}\right)\right]$, with $\bar{\tau}^{\varepsilon}=\inf \left\{t \geq 0: \bar{X}_{t}^{\varepsilon} \notin D_{0}\right\}$, provides another approximation of $v(x)$ (note that we use again a boundary shifting method).

Benchmarks are provided by the software FREEFEM with which we compute an approximation of $v(x)$ by a finite element method, using around $1.5 \times 10^{6}$ triangles and $7 \times 10^{5}$ vertices (finite elements basis consists of polynomial functions of order 1). Indeed we observe that with this space discretization order the finite element method is at convergence.

Table 1 shows the results. We provide $95 \%$ excess confidence intervals for $\mathbb{E}^{x}\left[f\left(\bar{X}_{\bar{\tau}}\right)\right]$ and $\mathbb{E}^{x}\left[f\left(\bar{X}_{\bar{\tau}^{\varepsilon}}^{\varepsilon}\right)\right]$. It seems that our Transformed Euler scheme converges quicker to the benchmark than the standard Euler scheme applied on regularized coefficients. Indeed we notice that the value at convergence provided by FreeFem tends to be in the confidence intervals for $\mathbb{E}^{x}\left[f\left(\bar{X}_{\bar{\tau}}\right)\right]$, while it is not the case for $\mathbb{E}^{x}\left[f\left(\bar{X}_{\bar{\tau}^{\varepsilon}}^{\varepsilon}\right)\right]$. 


\begin{tabular}{cccc}
\hline Point $x$ & $\begin{array}{c}\text { Finite Element } \\
\text { by FREEFEM } \\
\left(7.10^{5} \text { vertices }\right)\end{array}$ & $\begin{array}{c}\text { Euler Scheme on } \\
\text { regularized coefficients } \\
\left(h_{n}=10^{-n}, n=4,5,6\right)\end{array}$ & $\begin{array}{c}\text { Transformed } \\
\text { Euler Scheme } \\
\left(h_{n}=10^{-n}, n=2,4,5,6\right)\end{array}$ \\
\hline$x=(0,0.5)^{*}$ & -0.1207 & - & {$[-0.138615 ;-0.136341]$} \\
& & {$[-0.117842 ;-0.115607]$} & {$[-0.122637 ;-0.120408]$} \\
& & {$[-0.119072 ;-0.116820]$} & {$[-0.122415 ;-0.120183]$} \\
& {$[-0.119918 ;-0.117666]$} & {$[-0.121583 ;-0.119353]$} \\
\hline$x=(0.9,0.05)^{*}$ & 0.92527 & - & {$[0.825931 ; 0.827528]$} \\
& & {$[0.915922 ; 0.917244]$} & {$[0.923715 ; 0.924973]$} \\
& & {$[0.922148 ; 0.923478]$} & {$[0.924720 ; 0.926020]$} \\
& & {$[0.923188 ; 0.924518]$} & {$[0.924739 ; 0.926039]$} \\
\hline$x=(-0.3,-0.5)^{*}$ & -0.745461 & {$[-0.738013 ;-0.734145]$} & {$[-0.736531 ;-0.732709]$} \\
& & {$[-0.741029 ;-0.737169]$} & {$[-0.747555 ;-0.743703]$} \\
& & {$[-0.744675 ;-0.740819]$} & {$[-0.747759 ;-0.743746]$} \\
& &
\end{tabular}

Table 1: Approximated values of the solution $v(x)$ of $\left(\mathcal{E}_{\mathrm{T}, \text { bounded } D_{0}}^{0}\right)$ at points $x=$ $(0,0.5)^{*},(0.9,0.05)^{*},(-0.3,-0.5)^{*}$ computed with a finite element method $\left(7.10^{5}\right.$ vertices $)$, a standard Euler scheme applied on a regularisation $a^{\varepsilon}$ of $a$, and our tranformed Euler scheme (we provide confidence intervals for these two last values; we use $N=10^{6}$ Monte Carlo samples, and different values of $h_{n}$ ).

Example 2. We now turn to some parabolic example (with the same matrixvalued coefficient $a)$. We consider the following problem $\left(\mathcal{P}_{\mathrm{T}, \text { bounded }} D_{0}\right)$ :

$$
\left\{\begin{aligned}
\partial_{t} u(t, x)-\mathcal{L} u(t, x) & =0 & & \forall(t, x) \in(0, T] \times D \\
\left\langle a_{+} \nabla_{x} u_{+}(t, y)-a_{-} \nabla_{x} u_{-}(t, y), \nu(y)\right\rangle & =0 & & \forall(t, y) \in(0, T] \times \Gamma \quad(\star) \\
u(t, y+) & =u(t, y-) & & \forall(t, y) \in[0, T] \times \Gamma \\
u(t, x) & =0 & & \forall(t, x) \in(0, T] \times \partial D_{0} \\
u(0, x) & =u_{0}(x) & & \forall x \in \bar{D}_{0} .
\end{aligned}\right.
$$

Here we will take $T=0.1$ and

$$
u_{0}(x)=10 *\left(1-|x|^{2}\right) .
$$

Note that $u_{0}$ belongs to $H_{0}^{1}\left(D_{0}\right)$ and is therefore compatible with the uniform Dirichlet boundary condition in $\left(\mathcal{P}_{\mathrm{T}, \text { bounded }} D_{0}\right)$. But it does not belong to the domain $\mathcal{D}(A)$, as it does not satisfy the transmission condition $(\star)$.

Nevertheless one can hope that

$$
\mathbb{E}^{x}\left[u_{0}\left(\bar{X}_{t}\right) \mathbf{1}_{t \leq \bar{\tau}}\right] \underset{h_{n} \rightarrow 0}{\longrightarrow} u(t, x)
$$




\begin{tabular}{cccc}
\hline Point $x$ & $\begin{array}{c}\text { Finite Element } / \\
\text { Crank-Nicholson } \\
\left(4.5 \times 10^{5} \text { vertices, }\right. \\
300 \text { time steps })\end{array}$ & $\begin{array}{c}\text { Euler Scheme on } \\
\text { regularised coefficients } \\
\left(h_{n}=10^{-n}, n=4,5,6,7\right)\end{array}$ & $\begin{array}{c}\text { Transformed } \\
\text { Euler Scheme } \\
\left(h_{n}=10^{-n}, n=4,5,6,7\right)\end{array}$ \\
\hline$x=(0,0.5)^{*}$ & 2.26288 & & \\
& & - & - \\
& & {$[2.26438 ; 2.27807]$} & {$[2.2769 ; 2.29062]$} \\
& {$[2.25639 ; 2.27025]$} & {$[2.26869 ; 2.28255]$} \\
& {$[2.2554 ; 2.26926]$} & - \\
& & - & - \\
& & - & {$[2.25928 ; 2.27314]$} \\
\hline$x=(0.9,0.05)^{*}$ & & {$[0.266988 ; 0.27232]$} & {$[0.261169 ; 0.266501]$} \\
& {$[0.260363 ; 0.265695]$} & {$[0.256141 ; 0.261473]$} \\
\hline$x=(-0.3,-0.5)^{*}$ & 4.24525 & - & - \\
& & - & {$[4.231419 ; 4.245821]$} \\
& & {$[4.227519 ; 4.241921]$} & {$[4.237629 ; 4.252031]$} \\
\hline$x=(0,0.05)^{*}$ & 4.02857 & {$[4.232609 ; 4.247011]$} & - \\
& & - & {$[4.03105 ; 4.04655]$} \\
& & {$[4.026754 ; 4.042286]$} & {$[4.024784 ; 4.040316]$} \\
& & {$[4.017064 ; 4.032596]$} & {$[4.021594 ; 4.037126]$}
\end{tabular}

Table 2: Approximated values of the solution $u(T=0.1, x)$ of $\left(\mathcal{P}_{\mathrm{T}, \text { bounded } D_{0}}\right)$ at points $x=(0,0.5)^{*},(0.9,0.05)^{*},(-0.3,-0.5)^{*},(0,0.05)^{*}$ computed with a finite element / CrankNicholson scheme method $\left(4.5 \times 10^{5}\right.$ vertices, 300 times steps $)$, a standard Euler scheme applied on a regularisation $a^{\varepsilon}$ of $a$, and our tranformed Euler scheme (we provide confidence intervals for these two last values; we use $N=10^{6}$ Monte Carlo samples, and different values of $\left.h_{n}\right)$.

(here we use for example Theorem 4.4.5 in [20] and use again the notation $\bar{\tau}$ of Example 1).

Again we compute a Monte Carlo approximation of $\mathbb{E}^{x}\left[u_{0}\left(\bar{X}_{t}\right) \mathbf{1}_{t \leq \bar{\tau}}\right]$ on one side and of $\mathbb{E}^{x}\left[u_{0}\left(\bar{X}_{t}^{\varepsilon}\right) \mathbf{1}_{t \leq \bar{\tau}^{\varepsilon}}\right]$ on the other side (with $N=10^{6}$ paths and using again the boundary shifting method).

We use FREEFEM to compute an approximation of $u(t, x)$ by a finite element method (discretization in space) and a Crank-Nicholson scheme (discretization in time), using around $9 \times 10^{9}$ triangles and $4.5 \times 10^{5}$ vertices, and 300 time steps (we are at convergence).

Table 2 shows the results, for $t=T$. We provide again $95 \%$ excess confidence intervals, this time for $\mathbb{E}^{x}\left[u_{0}\left(\bar{X}_{t}\right) \mathbf{1}_{t \leq \bar{\tau}}\right]$ and $\mathbb{E}^{x}\left[u_{0}\left(\bar{X}_{t}^{\varepsilon}\right) \mathbf{1}_{t \leq \bar{\tau}^{\varepsilon}}\right]$. Again it seems that our transformed Euler scheme converges slightly quicker to the benchmark.

Example 3. Here we want to apply our scheme to a 3D example coming from Electromagnetism. In order to lighten the computations made by the 
PDE solver that we use as a benchmark (FreeFem again), we will focus on a stationary PDE.

Our example is inspired by the field of Electro- and Magnetoencephalography (EEG/MEG). We follow [44] for the exposure of the equations governing the electric potential distribution (vector field $\mathbf{E}$ ) and the resulting magnetic induction (vector field $\mathbf{B}$ ) generated in the brain.

These equations come from the quasistatic approximation of Maxwell's equations. When the permeability of the electric medium is that of the free space i.e. the permeability is constantly equal to $\mu_{0}$, these equations read

$$
\nabla \times \mathbf{E}=0, \quad \nabla \cdot \mathbf{E}=\frac{\rho}{\epsilon_{0}}
$$

for the electrical part and

$$
\nabla \times \mathbf{B}=\mu_{0} \mathbf{j}, \quad \nabla \cdot \mathbf{B}=0
$$

for the magnetic part. Here $\rho$ is the electric charge density, $\epsilon_{0}$ is the permittivity of free space and $\mathbf{j}$ represents the total electric current density (in addition $\nabla \times$ denotes the curl operator, applied to any vector field).

Here $\mathbf{j}$ is produced by neuronal activity and in bioelectromagnetism it is split into two contributions, i.e.

$$
\mathbf{j}=\mathbf{j}_{p}+\mathbf{j}_{s},
$$

where $\mathbf{j}_{p}$ and $\mathbf{j}_{s}$ are respectively the primary and the secondary current.

The secondary current is a passive current that is the result of the macroscopic electric field on charge carriers in the conducting medium. By Ohm's law one has

$$
a \mathbf{E}=\mathbf{j}_{s}
$$

where $a$ indicates the conductivity profile of the conductive medium.

Now, since the curl $\nabla \times \mathbf{E}$ equals zero, there exists a potential function $v$ such that $\mathbf{E}=-\nabla_{x} v$ and thus $-a \nabla_{x} v=\mathbf{j}_{s}$. Applying the divergence operator to the curl $\nabla \times \mathbf{B}$ one gets

$$
0=\nabla \cdot(\nabla \times \mathbf{B})=\nabla \cdot\left(\mu_{0} \mathbf{j}\right)=\mu_{0} \nabla \cdot\left(-a \nabla_{x} v+\mathbf{j}_{p}\right) .
$$

Hence, the electric potential function $v$ solves a Poisson equation of type

$$
\nabla \cdot\left(a \nabla_{x} v\right)=\nabla \cdot \mathbf{j}_{p} \text { in } D_{0} \subset \mathbb{R}^{3}
$$

where $D_{0}$ stands for the space volume of the conductive device.

In our example, we will consider that $D_{0}=(-5,5) \times(-5,5) \times(-5,5)$, that is $D_{0}$ is a 3 -dimensional cubic domain. We will consider that the medium inside $D_{0}$ is composed of two materials with different conductivity profiles, one to be found above the horizontal plane, the other one below. With our notations, $D_{+}=\left\{\left(x_{1}, x_{2}, x_{3}\right) \in D_{0}, x_{3}>0\right\}$ and $D_{-}=\left\{\left(x_{1}, x_{2}, x_{3}\right) \in D_{0}, x_{3}<0\right\}$. 
The conductivity coefficient is thus given by the matrix valued function

$$
a(x, y, z)=a_{+} \mathbb{I}_{z \geq 0}+a_{-} \mathbb{I}_{z<0}
$$

where we have chosen $a_{+}=\frac{1}{2}\left(\begin{array}{lll}1 & 1 & 1 \\ 1 & 2 & 2 \\ 1 & 2 & 3\end{array}\right)$ and $a_{-}=\frac{1}{2}\left(\begin{array}{ccc}1 & -1 & -1 \\ -1 & 2 & 0 \\ -1 & 0 & 3\end{array}\right)$.

From Gauss's reduction, for any $x=\left(x_{1}, x_{2}, x_{3}\right)^{*}$ we have $2 x^{*} a_{+} x=\left(x_{1}+x_{2}+x_{3}\right)^{2}+$ $\left(x_{2}+x_{3}\right)^{2}+x_{3}^{2}$ and $2 x^{*} a_{-} x=\left(x_{1}-x_{2}-x_{3}\right)^{2}+\left(x_{2}-x_{3}\right)^{2}+x_{3}^{2}$, which shows that $a_{+}$and $a_{-}$are symmetric positive definite matrices.

We assume that $\nabla \cdot \mathbf{j}_{p}=-1$ (this means that we assume that the primary current is smooth and non singular).

We thus aim at solving

$$
\left(\mathcal{E}_{\text {T,bounded } D_{0}}^{0 \text {,source } 1}\right)\left\{\begin{array}{rlrl}
-\mathcal{L} v(x) & =1 & & \forall x \in D \\
\left\langle a_{+} \nabla_{x} v_{+}(y)-a_{-} \nabla_{x} v_{-}(y), \nu(y)\right\rangle & =0 & & \forall y \in \Gamma \\
v(y+) & =v(y-) & \forall y \in \Gamma \\
v(x) & =0 & & \forall x \in \partial D_{0}
\end{array}\right.
$$

(note that we choose the uniform Dirichlet boundary condition while the uniform Neumann boundary condition is physically more relevant, cf [44]; up to our knowledge performing efficient stochastic numerical schemes for Neumann boundary value problems remains a challenging problem, even in the case of smooth coefficients, cf for example [34]).

In order to perform our stochastic numerical scheme one first notice that

$$
\sigma_{+}=\left(\begin{array}{ccc}
1 & 0 & 0 \\
1 & 1 & 0 \\
1 & 1 & 1
\end{array}\right), \quad \sigma_{-}=\left(\begin{array}{ccc}
1 & 0 & 0 \\
-1 & 1 & 0 \\
-1 & -1 & 1
\end{array}\right)
$$

satisfy $2 a_{+}=\sigma_{+} \sigma_{+}^{*}$ and $2 a_{-}=\sigma_{-} \sigma_{-}^{*}$. Here we have clearly $\partial a \equiv 0$.

Besides the co-normal vector fields are constant with

$$
\gamma_{+}(x)=\gamma_{+}=(1,2,3)^{*}, \gamma_{-}(x)=\gamma_{-}=(1,0,-3)^{*} .
$$

In particular, for any $\left(x_{1}, x_{2}, x_{3}\right)^{*} \in D_{-}$, we check that the projection decomposition along $\gamma^{+}$simplifies to

$$
\left(x_{1}, x_{2}, x_{3}\right)^{*}=\left(x_{1}-\frac{x_{3}}{3}, x_{2}-\frac{2 x_{3}}{3}, 0\right)^{*}+\frac{x_{3}}{3}(1,2,3)^{*}
$$

which shows that $F^{\gamma_{+}}\left(\left(x_{1}, x_{2}, x_{3}\right)^{*}\right)=x_{3} / 3$ and

$$
\pi_{\Gamma}^{\gamma_{+}}\left(\left(x_{1}, x_{2}, x_{3}\right)^{*}\right)=\left(x_{1}-\frac{x_{3}}{3}, x_{2}-\frac{2 x_{3}}{3}, 0\right)^{*} .
$$




\begin{tabular}{|c|c|c|}
\hline Point $x$ & $\begin{array}{c}\text { Finite Element } \\
\left(3.5 \times 10^{6} \text { vertices }\right)\end{array}$ & $\begin{array}{c}\text { Transformed Euler Scheme } \\
\quad\left(h_{n}=10^{-n}, n=3,4,5\right) \\
\text { estimated mean and stand. dev. (sd) }\end{array}$ \\
\hline \multirow{3}{*}{$x=(0,0,0.05)^{*}$} & 6.13578 & 6.14868 (sd: 4.25$)$ \\
\hline & & 6.154155 (sd: 4.257$)$ \\
\hline & & $6.15108(\mathrm{sd}: 4.25)$ \\
\hline \multirow{3}{*}{$x=(0,0,-0.1)^{*}$} & 6.1138 & 6.127855 (sd: 4.247449$)$ \\
\hline & & 6.134545 (sd: 4.258$)$ \\
\hline & & 6.136085 (sd: 4.268$)$ \\
\hline \multirow[t]{3}{*}{$x=(2,2,1)^{*}$} & 4.81464 & 4.84222 (sd: 3.82653$)$ \\
\hline & & $4.83942(\mathrm{sd}: 3.811)$ \\
\hline & & 4.83677 (sd: 3.816$)$ \\
\hline \multirow[t]{3}{*}{$x=(2,2,4.75)^{*}$} & 0.657751 & 0.6620455 (sd: 2.09) \\
\hline & & 0.655707 (sd: 2.083 ) \\
\hline & & 0.6576685 (sd: 2.09$)$ \\
\hline
\end{tabular}

Table 3: Approximated values of the solution $v(x)$ of $\left(\mathcal{E}_{\mathrm{T}, \text { bounded } D}^{0 \text {, source }}\right)$ at points $x=$ $(0,0,0.05)^{*},(0,0,-0.1)^{*},(2,2,1)^{*}$ and $(2,2,4.75)^{*}$, computed with a finite element method $\left(3.4 \times 10^{6}\right.$ vertices), and our tranformed Euler scheme (with $N=10^{6}$ Monte Carlo samples, and different values of $h_{n}$ ).

Similarly for any $\left(x_{1}, x_{2}, x_{3}\right)^{*} \in D_{+}$the projection decomposition along $\gamma^{-}$ reads

$$
\left(x_{1}, x_{2}, x_{3}\right)^{*}=\left(x_{1}+\frac{x_{3}}{3}, x_{2}, 0\right)^{*}-\frac{x_{3}}{3}(1,0,-3)^{*}
$$

which shows that $F^{\gamma-}\left(\left(x_{1}, x_{2}, x_{3}\right)^{*}\right)=-x_{3} / 3$ and

$$
\pi_{\Gamma}^{\gamma_{-}}\left(\left(x_{1}, x_{2}, x_{3}\right)^{*}\right)=\left(x_{1}+\frac{x_{3}}{3}, x_{2}, 0\right)^{*} .
$$

Thus it is easy to compute at each time step $\pi_{\Gamma}^{\gamma_{ \pm}}\left(\hat{X}_{t_{k+1}}\right)$ and $F^{\gamma_{ \pm}}\left(\hat{X}_{t_{k+1}}\right)$ and again we have everything in hand to perform our Tranformed Euler Scheme $\bar{X}$.

Table 3 shows the results.

To demand a very fine space discretization in FreeFem is computationally very costly and we have not been able to achieve such computations on the machines at our disposal. Consequently we are not sure that the result given by FreeFem is at convergence (the number of vertices indicated in Table 3 may seem high, but in fact this corresponds to a quite coarse discretization of the cube $D_{0}=(-5,5)^{3}$, as we are in $3 \mathrm{D}$; the error is expected to be of order $\left.10^{-1}\right)$.

Thus when testing the numerical stochastic scheme we have limited ourselves to $h_{n}=10^{-5}$ (also $h_{n}=10^{-5}$ was already time demanding, on this example). Also we have not computed confidence intervals, as we are not sure that the benchmark provided by FreeFem is totally relevant.

Note though, that the results presented in Table 3 show some coherence: it seems that both methods tend to converge to a common value. 


\section{Acknowledgement}

Research partially supported by Labex Bézout (for Miguel Martinez).

\section{References}

[1] Aronson, D.G., 1967. Bounds for the fundamental solution of a parabolic equation. Bull. Amer. Math. Soc. 73, 890896. URL: https://doi.org/10.1090/S0002-9904-1967-11830-5, doi:10.1090/S0002-9904-1967-11830-5.

[2] Bally, V., Talay, D., 1995. The Euler scheme for stochastic differential equations: error analysis with Malliavin calculus, volume 38, pp. 35-41. URL: https://doi-org-s.fennec.u-pem.fr/10.1016/0378-4754(93)E0064-C, doi:10.1016/0378-4754(93)E0064-C. probabilités numériques (Paris, 1992).

[3] Bokil, V.A., Gibson, N.L., Nguyen, S.L., Thomann, E.A., Waymire, E.C., 2020. An Euler-Maruyama method for diffusion equations with discontinuous coefficients and a family of interface conditions. J. Comput. Appl. Math. 368, 112545, 18. URL: https://doi-org-s.fennec.u-pem.fr/10.1016/j.cam.2019.112545, doi:10.1016/j.cam.2019.112545.

[4] Bossy, M., Champagnat, N., Maire, S., Talay, D., 2010. Probabilistic interpretation and random walk on spheres algorithms for the PoissonBoltzmann equation in molecular dynamics. M2AN Math. Model. Numer. Anal. 44, 997-1048. URL: http://dx.doi.org/10.1051/m2an/2010050, doi:10.1051/m2an/2010050.

[5] Bossy, M., Gobet, E., Talay, D., 2004. A symmetrized Euler scheme for an efficient approximation of reflected diffusions. J. Appl. Probab. 41, 877-889.

[6] Brezis, H., 1983. Analyse fonctionnelle. Collection Mathématiques Appliquées pour la Maîtrise. [Collection of Applied Mathematics for the Master's Degree], Masson, Paris. Théorie et applications. [Theory and applications].

[7] De Giorgi, E., 1957. Sulla differenziabilità e l'analiticità delle estremali degli integrali multipli regolari. Mem. Accad. Sci. Torino. Cl. Sci. Fis. Mat. Nat. (3) 3, 25-43.

[8] Dereudre, D., Mazzonetto, S., Roelly, S., 2016. Exact simulation of brownian diffusions with drift admitting jumps.

[9] Étoré, P., 2006. On random walk simulation of one-dimensional diffusion processes with discontinuous coefficients. Electron. J. Probab. 11, no. 9, 249-275. URL: http://dx.doi.org/10.1214/EJP.v11-311, doi:10.1214/EJP.v11-311. 
[10] Étoré, P., Lejay, A., 2007. A Donsker theorem to simulate one-dimensional processes with measurable coefficients. ESAIM Probab. Stat. 11, 301-326. URL: http://dx. doi .org/10.1051/ps : 2007021, doi:10.1051/ps:2007021.

[11] Étoré, P., Martinez, M., 2013. Exact simulation of one-dimensional stochastic differential equations involving the local time at zero of the unknown process. Monte Carlo Methods Appl. 19, 41-71. URL: http://dx.doi.org/10.1515/mcma-2013-0002, doi:10.1515/mcma-20130002 .

[12] Étoré, P., Martinez, M., 2014. Exact simulation for solutions of one-dimensional stochastic differential equations with discontinuous drift. ESAIM Probab. Stat. 18, 686-702. URL: http://dx.doi.org/10.1051/ps/2013053, doi:10.1051/ps/2013053.

[13] Étoré, P., Martinez, M., 2017. Time inhomogeneous stochastic differential equations involving the local time of the unknown process, and associated parabolic operators. Stochastic Processes and their Applications URL: https://hal .archives-ouvertes.fr/hal-01356270, doi:https://doi.org/10.1016/j.spa.2017.09.018.

[14] Étoré, P., Martinez, M., 2020. Stochastic processes associated to multidimensional parabolic transmission problems in divergence form. Preprint

[15] Friedman, A., 1964. Partial differential equations of parabolic type. Prentice-Hall Inc., Englewood Cliffs, N.J.

[16] Frikha, N., 2018. On the weak approximation of a skew diffusion by an euler-type scheme. Bernoulli 24, 1653-1691. URL: https://doi.org/10.3150/16-BEJ909, doi:10.3150/16-BEJ909.

[17] Fukushima, M., Oshima, Y., Takeda, M., 2011. Dirichlet forms and symmetric Markov processes. volume 19 of De Gruyter Studies in Mathematics. extended ed., Walter de Gruyter \& Co., Berlin.

[18] Gilbarg, D., Trudinger, N.S., 1983. Elliptic partial differential equations of second order. Grundlehren der mathematischen Wissenschaften, Springer-Verlag, Berlin, New York. URL: http://opac.inria.fr/record=b1091868. cataloging based on CIP information.

[19] Gobet, E., 2001. Euler schemes and half-space approximation for the simulation of diffusion in a domain. ESAIM Probab. Statist. 5, 261-297. URL: https://doi.org/10.1051/ps:2001112, doi:10.1051/ps:2001112.

[20] Gobet, E., 2016. Monte-Carlo methods and stochastic processes. CRC Press, Boca Raton, FL. From linear to non-linear. 
[21] Karatzas, I., Shreve, S.E., 1991. Brownian motion and stochastic calculus. volume 113 of Graduate Texts in Mathematics. Second ed., Springer-Verlag, New York. URL: http://dx.doi.org/10.1007/978-1-4612-0949-2, doi:10.1007/978-1-4612-0949-2.

[22] Kloeden, P.E., Platen, E., 1992. Numerical solution of stochastic differential equations / Peter E. Kloeden, Eckhard Platen. Springer-Verlag, Berlin ; New York :.

[23] Ladyženskaja, O.A., Solonnikov, V.A., Ural'ceva, N.N., 1967. Linear and Quasi Linear Equations of Parabolic type. Izdat. "Nauka", Moscow.

[24] Ladyzhenskaya, O.A., Rivkind, V.Y., Ural'tseva, N.N., 1966. The classical solvability of diffraction problems. Boundary value problems of mathematical physics. Part 4 92, 116-146.

[25] Le Gall, J.F., 1984. One-dimensional stochastic differential equations involving the local times of the unknown process, in: Stochastic analysis and applications (Swansea, 1983). Springer, Berlin. volume 1095 of Lecture Notes in Math., pp. 51-82. URL: https://dx-doi-org.fennec.u-pem.fr/10.1007/BFb0099122, doi:10.1007/BFb0099122.

[26] Lejay, A., 2006. On the constructions of the skew Brownian motion. Probab. Surv. 3, 413-466. URL: $\quad$ http://dx.doi.org/10.1214/154957807000000013, doi:10.1214/154957807000000013.

[27] Lejay, A., Lenôtre, L., Pichot, G., 2019. An exponential timestepping algorithm for diffusion with discontinuous coefficients. Journal of Computational Physics 396. doi:10.1016/j.jcp.2019.07.013.

[28] Lejay, A., Maire, S., 2013. New monte carlo schemes for simulating diffusions in discontinuous media. Journal of Computational and Applied Mathematics 245, 97?116. doi:10.1016/j.cam.2012.12.013.

[29] Lejay, A., Martinez, M., 2006. A scheme for simulating one-dimensional diffusion processes with discontinuous coefficients. Ann. Appl. Probab. 16, 107-139. URL: https://dx-doi-org.fennec.u-pem.fr/10.1214/105051605000000656, doi:10.1214/105051605000000656.

[30] Lenôtre, L., 2015. étude et simulation des processus de diffusion biaisés. Ph. D Thesis .

[31] Lieberman, G., 1996. Second Order Parabolic Differential Equations. World Scientific. URL: https://books . google.fr/books?id=s9Guiwylm3cC. 
[32] Limic, N., 2011. Markov jump processes approximating a nonsymmetric generalized diffusion. Applied Mathematics and Optimization 64, 101-133. URL: http://dx.doi.org/10.1007/s00245-011-9133-1, doi:10.1007/s00245-011-9133-1.

[33] Lions, J.L., Magenes, E., 1972. Non-homogeneous boundary value problems and applications. Vol. I. Springer-Verlag, New York-Heidelberg. Translated from the French by P. Kenneth, Die Grundlehren der mathematischen Wissenschaften, Band 181.

[34] Maire, S., Tanré, E., 2013. Monte Carlo approximations of the Neumann problem. Monte Carlo Methods and Applications 19, 201-236. URL: https://hal .inria.fr/hal-00677529, doi:10.1515/mcma-2013-0010.

[35] Martinez, M., 2004. Inbterprétations probabilistes d'opérateurs sous forme divergence et analyse des méthodes numériques probabilistes associées. $\mathrm{Ph}$. D Thesis .

[36] Martinez, M., Talay, D., 2006. Discrétisation d'équations différentielles stochastiques unidimensionnelles à générateur sous forme divergence avec coefficient discontinu. C. R. Math. Acad. Sci. Paris 342, 51-56. URL: https://dx-doi-org.fennec.u-pem.fr/10.1016/j.crma.2005.10.025, doi:10.1016/j.crma.2005.10.025.

[37] Martinez, M., Talay, D., 2012. One-dimensional parabolic diffraction equations: pointwise estimates and discretization of related stochastic differential equations with weighted local times. Electron. J. Probab. 17, no. 27, 30. URL: https://dx-doi-org.fennec.u-pem.fr/10.1214/EJP.v17-1905, doi:10.1214/EJP.v17-1905.

[38] McLean, W., 2000. Strongly Elliptic Systems and Boundary Integral Equations. Cambridge University Press. URL: https://books.google.ca/books?id=RILqjEeMfKOC.

[39] Moser, J., 1963. A Harnack inequality for parabolic differential equations, in: Outlines Joint Sympos. Partial Differential Equations (Novosibirsk, 1963). Acad. Sci. USSR Siberian Branch, Moscow, pp. 343-347.

[40] Moser, J., 1964. A Harnack inequality for parabolic differential equations. Comm. Pure Appl. Math. 17, 101134. URL: https://doi.org/10.1002/cpa.3160170106, doi:10.1002/cpa.3160170106.

[41] Moser, J., 1967. Correction to: "A Harnack inequality for parabolic differential equations". Comm. Pure Appl. Math. 20, 231-236. URL: https://doi.org/10.1002/cpa.3160200107, doi:10.1002/cpa.3160200107.

[42] Nash, J., 1957. Parabolic equations. Proc. Nat. Acad. Sci. U.S.A. 43, $754-758$. 
[43] Nash, J., 1958. Continuity of solutions of parabolic and elliptic equations. Amer. J. Math. 80, 931-954. URL: https://doi.org/10.2307/2372841, doi: $10.2307 / 2372841$.

[44] Piastra, M.C., Nüßing, A., Vorwerk, J., Bornfleth, H., Oostenveld, R., Engwer, C., Wolters, C.H., 2018. The discontinuous galerkin finite element method for solving the meg and the combined meg/eeg forward problem. Frontiers in Neuroscience 12, 30. URL: https://www.frontiersin.org/article/10.3389/fnins.2018.00030, doi:10.3389/fnins.2018.00030.

[45] Stroock, D.W., 1988. Diffusion semigroups corresponding to uniformly elliptic divergence form operators, in: Séminaire de Probabilités, XXII. Springer, Berlin. volume 1321 of Lecture Notes in Math., pp. 316-347. URL: http://dx.doi.org/10.1007/BFb0084145, doi:10.1007/BFb0084145.

[46] Stroock, D.W., Zheng, W., 1997. Markov chain approximations to symmetric diffusions. Annales de l'I.H.P. Probabilités et statistiques 33, 619-649. URL: http://eudml .org/doc/77584.

[47] Talay, D., Tubaro, L., 1990. Expansion of the global error for numerical schemes solving stochastic differential equations. Stochastic Anal. Appl. 8, 483-509 (1991). URL: https://doi-org-s.fennec.u-pem.fr/10.1080/07362999008809220, doi:10.1080/07362999008809220. 